\title{
Euclid Near Infrared Spectrometer and Photometer instrument concept and first test results obtained for different breadboards models at the end of phase C
}

Maciaszek, Thierry; Ealet, Anne; Jahnke, Knud; Prieto, Eric; Barbier, Rémi ; Mellier, Yannick ; Beaumont, Florent; Bon, William ; Bonefoi, Anne ; Carle, Michael

Total number of authors:

16

Published in:

Space Telescopes and Instrumentation 2016: Optical, Infrared, and Millimeter Wave

Link to article, DOI:

$10.1117 / 12.2232941$

Publication date:

2016

Document Version

Publisher's PDF, also known as Version of record

Link back to DTU Orbit

Citation (APA):

Maciaszek, T., Ealet, A., Jahnke, K., Prieto, E., Barbier, R., Mellier, Y., Beaumont, F., Bon, W., Bonefoi, A., Carle, M., Toulouse-Aastrup, C., Andersen, M. I., Sørensen, A. N., Jakobsen, P., Hornstrup, A., \& Jessen, N. C. (2016). Euclid Near Infrared Spectrometer and Photometer instrument concept and first test results obtained for different breadboards models at the end of phase C. In H. A. MacEwen, G. G. Fazio, \& M. Lystrup (Eds.), Space Telescopes and Instrumentation 2016: Optical, Infrared, and Millimeter Wave (Vol. 9904). [99040T] SPIE International Society for Optical Engineering. https://doi.org/10.1117/12.2232941

\section{General rights}

Copyright and moral rights for the publications made accessible in the public portal are retained by the authors and/or other copyright owners and it is a condition of accessing publications that users recognise and abide by the legal requirements associated with these rights.

- Users may download and print one copy of any publication from the public portal for the purpose of private study or research.

- You may not further distribute the material or use it for any profit-making activity or commercial gain

- You may freely distribute the URL identifying the publication in the public portal 


\title{
Euclid Near Infrared Spectrometer and Photometer instrument concept and first test results obtained for different breadboards models at the end of phase $C$
}

\author{
Thierry Maciaszek: Ctr. National d'Études Spatiales, and LAM (Laboratoire d'Astrophysique d'Astrophysique de Marseille) UMR 7326 (France) \\ Anne Ealet: Ctr. de Physique des Particules de Marseille (France) \\ Knud Jahnke: Max-Planck-Institut für Astronomie (Germany) \\ Eric Prieto: Aix Marseille Université, CNRS, LAM (Laboratoire d'Astrophysique de Marseille) UMR 7326 (France) \\ Rémi Barbier: Institut de Physique Nucléaire de Lyon (France) \\ Yannick Mellier: Institut d'Astrophysique de Paris (France), and Commissariat à l'Énergie Atomique (France) \\ Florent Beaumont, William Bon, Anne Bonefoi, Michael Carle, Amandine Caillat, Anne Costille, Doriane Dormoy, Franck Ducret, Christophe Fabron, \\ Aurélien Febvre, Benjamin Foulon, Jose Garcia, Jean-Luc Gimenez, Emmanuel Grassi, Philippe Laurent, David Le Mignant, Laurent Martin, Christelle \\ Rossin, Tony Pamplona, Patrice Sanchez, Sebastien Vives: Aix Marseille Université, CNRS, LAM (Laboratoire d'Astrophysique de \\ Marseille) UMR 7326, Marseille, (France) \\ Jean Claude Clémens, William Gillard, Mathieu Niclas, Aurélia Secroun, Benoit Serra: Ctr de Physique des Particules de Marseille (France) \\ Bogna Kubik, Sylvain Ferriol: Institut de Physique Nucléaire de Lyon (France) \\ Jérome Amiaux, Jean Christophe Barrière, Michel Berthe: Commissariat à l'Énergie Atomique (France) \\ Cyrille Rosset: Laboratoire Astroparticule et Cosmologie (France) \\ Juan Francisco Macias-Perez : Laboratoire de Physique Subatomique et Cosmologie (France) \\ Natalia Auricchio, Adriano De Rosa, Enrico Franceschi, Gian Paolo Guizzo, Gianluca Morgante, Francesca Sortino, Massimo Trifoglio, Luca \\ Valenziano: INAF - IASF Bologna (Italy) \\ Laura Patrizii, T. Chiarusi, F. Fornari, F. Giacomini, A. Margiotta, N. Mauri, L. Pasqualini, G. Sirri, M. Spurio, M. Tenti, R. Travaglini: INFN Bologna \\ (Italy) \\ Stefano Dusini, F. Dal Corso, F. Laudisio, C. Sirignano, L.Stanco, S.Ventura, Enrico Borsato: INFN Padova (Italy) \\ Carlotta Bonoli, Favio Bortoletto, Andrea Balestra, Maurizio D'Alessandro, Eduardo MedinaCeli, Ruben Farinelli: INAF - Osservatorio Astronimico di \\ Padova (Italy) \\ Leonardo Corcione, Sebastiano Ligori: INAF - Observatorio Astronomico di Torino (Italy) \\ Frank Grupp, Carolin Wimmer: Max-Planck-Institut für extraterrestrische Physik (Germany) \\ Felix Hormuth, Gregor Seidel, Stefanie Wachter: Max-Planck-Institut für Astronomie (Germany) \\ Cristobal Padilla, Mikel Lamensans: Institut de Física d'Altes Energies (IFAE) (Spain) \\ Ricard Casas, Ivan Lloro: Institut de Ciències de l'Espai, IEEC-CSIC (Spain) \\ Rafael Toledo-Moreo, Jaime Gomez, Carlos Colodro-Conde, David Lizán; Space Science and Engineering Lab (SSEL), Universidad Politécnica de \\ Cartagena (Spain) \\ Jose Javier. Diaz; Instituto de Astrofisica de Canarias (Spain) \\ Per B. Lilje: University of Oslo (Norway) \\ Corinne Toulouse-Aastrup, Michael I. Andersen, Anton N. Sørensen, Peter Jakobsen: Dark Cosmology Centre, Niels Bohr Institute, Copenhagen \\ University (Denmark) \\ Allan Hornstrup, Niels-Christian Jessen: DTU Space, Denmark \\ Cédric Thizy: Université de Liège - ULg CSL (Centre Spatial de Liège) \\ Warren Holmes, Ulf Israelsson, Michael Seiffert, Augustyn Waczynski: NASA (USA) \\ René J. Laureijs, Giuseppe Racca, Jean-Christophe Salvignol, Tobias Boenke, Paolo Strada; European Space Agency/ESTEC
}

On behalf of the Euclid Consortium

\begin{abstract}
The Euclid mission objective is to understand why the expansion of the Universe is accelerating through by mapping the geometry of the dark Universe by investigating the distance-redshift relationship and tracing the evolution of cosmic structures. The Euclid project is part of ESA's Cosmic Vision program with its launch planned for 2020 (ref [1]).

The NISP (Near Infrared Spectrometer and Photometer) is one of the two Euclid instruments and is operating in the near-IR spectral region (900$2000 \mathrm{~nm}$ ) as a photometer and spectrometer. The instrument is composed of:

- a cold (135K) optomechanical subsystem consisting of a Silicon carbide structure, an optical assembly (corrector and camera lens), a filter wheel mechanism, a grism wheel mechanism, a calibration unit and a thermal control system

- a detection subsystem based on a mosaic of 16 HAWAII2RG cooled to $95 \mathrm{~K}$ with their front-end readout electronic cooled to $140 \mathrm{~K}$, integrated on a mechanical focal plane structure made with molybdenum and aluminum. The detection subsystem is mounted on the optomechanical subsystem structure

- a warm electronic subsystem (280K) composed of a data processing / detector control unit and of an instrument control unit that interfaces with the spacecraft via a 1553 bus for command and control and via Spacewire links for science data

This presentation describes the architecture of the instrument at the end of the phase C (Detailed Design Review), the expected performance, the technological key challenges and preliminary test results obtained for different NISP subsystem breadboards and for the NISP Structural and Thermal model (STM).
\end{abstract}

Keywords: Euclid, Spectroscopy, Photometry, Infrared, Instrument, NISP

Space Telescopes and Instrumentation 2016: Optical, Infrared, and Millimeter Wave, edited by

Howard A. MacEwen, Giovanni G. Fazio, Makenzie Lystrup, Proc. of SPIE Vol. 9904

99040T - (C) 2016 SPIE · CCC code: 0277-786X/16/\$18 - doi: 10.1117/12.2232941

Proc. of SPIE Vol. 9904 99040T-1 


\section{INTRODUCTION}

Euclid is a wide-field space mission concept dedicated to the high-precision study of dark energy and dark matter. Euclid will carry out an imaging and spectroscopic wide survey of the entire extra-galactic sky $\left(15000 \mathrm{deg}^{2}\right)$ along with a deep survey covering at least $40 \mathrm{deg}^{2}$. To achieve these science objectives, the current Euclid reference design consists of a wide field telescope to be placed in L2 orbit by a Soyuz launch with a 6 years' mission lifetime. The payload consists of a $1.2 \mathrm{~m}$ diameter 3-mirror telescope with two channels: a VISible imaging channel (VIS) and a Near Infrared Spectrometer and Photometer channel (NISP). Both instruments observe simultaneously the same Field of View (FoV) on the sky and the system design is optimized for a sky survey in a step-and-stare tiling mode.

The NISP Instrument is operating in the $920-2000 \mathrm{~nm}$ range at a temperature lower than $140 \mathrm{~K}$, except for detectors, which are cooled down to $\sim 95 \mathrm{~K}$ or below. The warm electronics will be located in the service module, at room temperature (around $20^{\circ} \mathrm{C}$ ).

The NISP instrument has two main observing modes: the photometric mode, for the acquisition of images with broad band filters, and the spectroscopic mode, for the acquisition of slitless dispersed images on the detectors.

In the photometer mode the NISP instrument images the telescope light in the wavelength range from 920nm to 2000nm (Y, J, H bands). The spatial sampling is required to be $0.3 \mathrm{arcsec}$ per pixel. The FoV of the instrument is $0.55 \mathrm{deg}^{2}$ having a rectangular shape of $0.763 \mathrm{deg} \times 0.722 \mathrm{deg}$.

In the spectrometer mode the light of the observed target is dispersed by means of grisms covering the wavelength range of $950-1850 \mathrm{~nm}$. In order to provide a flat resolution over the specified wavelength range, four grisms are mounted in a wheel. These four grisms yield three dispersion directions tilted against each other by $90^{\circ}$ in order to reduce confusion from overlapping (due to slitless observing mode). The field and waveband definitions used in the individual configurations for spectroscopy and photometry are:

- $\quad$ Three photometric bands:

1. Y Band: $950-1192 \mathrm{~nm}$

2. J Band: $1192-1544 \mathrm{~nm}$

3. H Band: $1544-2000 \mathrm{~nm}$

- $\quad$ Four Slitless spectroscopic bands:

1. Red $0^{\circ} ; 90^{\circ}$ and $180^{\circ}$ dispersion: $1250-1850 \mathrm{~nm}$

2. Blue $0^{\circ}$ dispersion: $920-1300 \mathrm{~nm}$

The spectral resolution shall be higher than 250 for a one arcsec homogenous illumination object size. For such an object, the flux limit in spectroscopy shall be lower than $2 \times 10^{-16} \mathrm{erg} \cdot \mathrm{cm}^{-2} \cdot \mathrm{s}^{-1}$ at $1600 \mathrm{~nm}$ wavelength. As with all slitless spectrographs, the real resolution varies with the object size (the smaller the size is, higher the resolution is).

The image quality of the instrument in flight shall deliver a $50 \%$ radius encircled energy better than $0.3 \operatorname{arcsec}$ and a $80 \%$ one better than 0.7 arcsec. There is a variation due to diffraction with wavelength.

The NISP budgets are presently the following:

The instrument sits in a box of $1.0 \times 0.6 \times 0.5 \mathrm{~m}$

The total mass of the instrument is $155 \mathrm{~kg}$

The maximum power consumption is $178 \mathrm{~W}$

The instrument will produce 290GBit of data per day

European Contributor countries for NISP are: France, Italy, Germany, Spain, Denmark and Norway, ESA for the engineering detectors and USA (NASA) for the flight detectors.

\section{NISP GLOBAL DESCRIPTION}

The NISP instrument consists of three main Assemblies

- The NI-OMA (Opto-Mechanical Assembly), composed of the Mechanical Support Structure (NI-SA) and its thermal control (NI-TC), the Optical elements (NI-OA), the Filter Wheel Assembly (NI-FWA), the Grism Wheel Assembly (NI-GWA), the Calibration Unit (NI-CU). The NI-OMA structure supports the Optical elements, the calibration unit, the Filter and Grism Wheel Units and the detection system. It provides the thermo-mechanical interface towards the Euclid PLM.

- The NI-DS (Detector System Assembly) is composed by the Focal Plane Assembly (NI-FPA; the mechanical part of NI-DS) and by the Sensor Chip System (NI-SCS) compose). The NI-DS comprises the 16 H2RG detectors and associated 16 ASICS (Sidecars), passively cooled at operating temperature $(<100 \mathrm{~K}$ for the detectors; $140 \mathrm{~K}$ for the ASICS Sidecar). Thermal stabilization of the detector is "naturally" obtained thanks to the very good thermal stability provided by the Euclid PLM at the NISP interfaces

- The Warm Electronics Assembly (NI-WE), composed of the Instrument Data Processing Unit and Control Unit (NI-DPU/DCU), and the Instrument Control Unit (NI-ICU). The NI-ICU is managing the commanding and the control of the instrument. It is interfaced with the satellite via a 1553 bus. The NI-DPU/DCU controls the Sensor Chip System and basic image processing such as co-adding (DCU function) and the science onboard data processing, the compression and transfer of scientific data to the S/C Mass Memory using Spacewire links (DPU function). The NI-DPU/DCU functions are regrouped in a single mechanical box for controlling eight detectors. There are two NIDPU/DCU boxes.

The NI-DS is screwed on the NI-OMA ( $\mathrm{SiC}$ panel to SiC panel). The NI-OMA+NI-DS is located in the Euclid spacecraft Payload module in a cold environment (130K). The Warm electronic are located in the Euclid spacecraft Service Module at room temperature. A dedicated harness interconnects the NI-OMA, the NI-DS, the NI-WE and different spacecraft electronics boxes 

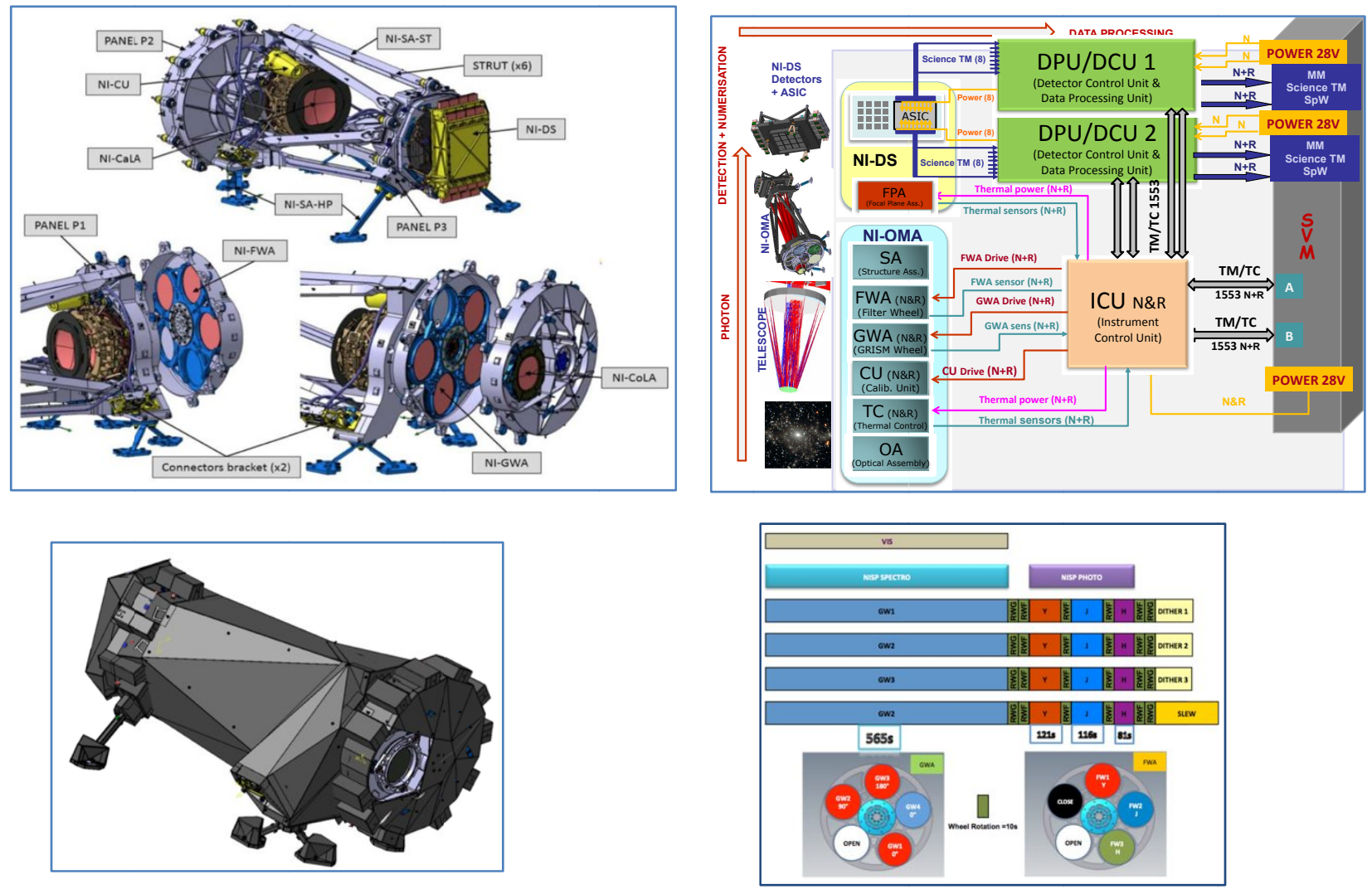

Figure 2-1: NISP global overview and functional scheme

The instrument has a specific observing sequence that will be repeated throughout the mission lifetime.

The survey is decomposed into fields. To avoid confusion and increase spatial resolution, each field is observed with 4-dithered frames for each band. Only one grism will be observed at each dither. The spectrum confusion is minimized thanks to the $90^{\circ}$ rotation of the dispersion within to same spectral band. During the nominal survey, only the three red grisms are used. Meanwhile, the blue grism is used only during the deep survey. Each sequence of exposures will consist of a $565 \mathrm{~s}$ spectroscopic observation followed by 80-120s of photometric observations in each of the $\mathrm{Y}, \mathrm{J}, \mathrm{H}$ photometric bands. The Filter and the Grism wheels are activated between each observation to set the instrument in the proper configuration. Due to the specificity of the H2RG detector, no shutter is needed to stop the integration. The shutter is included for dark calibration purposes (close position of the filter wheel) to prevent accumulation of persistence during satellite slews.

\section{NISP OPTO-MECHANICAL ASSEMBLY (NI-OMA)}

The structure (NI-SA, see ref [2]):

The structure will be made of Silicon Carbide ( $\mathrm{SiC}$ ). The choice of such material has been the result of a long trade-off among carbon fiber, aluminum and $\mathrm{SiC}$, mainly. The main driver was the very tight constraint of dimension stability of the system from AIV to the end of the mission.
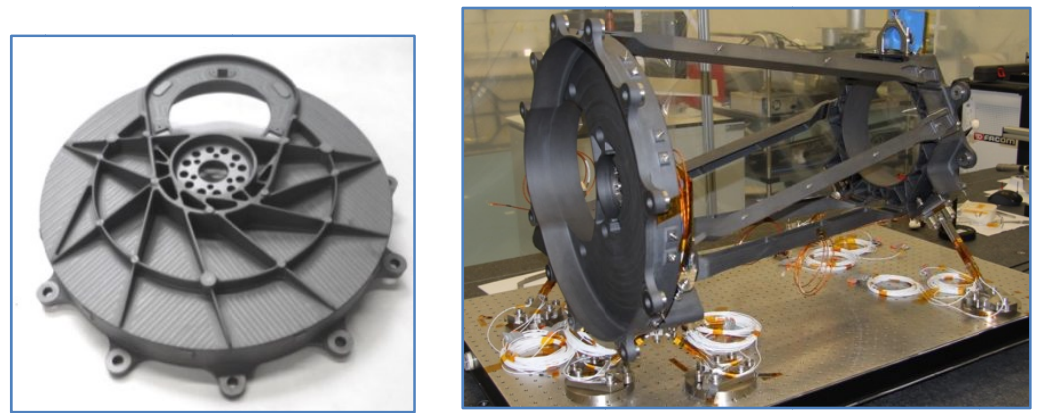

Figure 3-1: NISP Structure (STM) 


\section{The thermal control (NI-TC):}

Operating temperature and thermal stability of the units are key drivers for the instrument. The structure (NI-SA) is by design thermally insulated from the Payload module (PLM) baseplate through Invar bipods and monopods. The total conductance from NI-OMA to the Baseplate is around $0.035 \mathrm{~W} / \mathrm{K}$ which minimizes the transferred heat between these elements. In this configuration, the units inside the NI-OMA, and especially the optical lenses, are less sensitive to fluctuations and can exploit the whole instrument thermal mass to operate in a more stable condition. Cooling down to the required operating temperatures is performed, mainly, by heat extraction through two conductive thermal interfaces provided by the PLM located on the NI-DS. The structure is entirely made of $\mathrm{SiC}$ which, given the good thermal conductivity of such material, ensures good temperature uniformity and an efficient heat extraction.

This NI-OMA is operated at a temperature around $130 \mathrm{~K}$ with a stability better than $0.3 \mathrm{~K}$ for the full mission operation, from ground to end of life. Radiative loads from the PLM cavity environment are efficiently shielded by an MLI shroud that surrounds the whole instrument. Both blanket surfaces, internal and external, are black Kapton to minimize straylight contamination.

The Figure below shows the results of the NISP FEM thermal model with the external interfaces in warm conditions and units nominal dissipation. The NI-SA temperature is around $132 \mathrm{~K}$ with an overall thermal gradient below $2 \mathrm{~K}$ and the optical lenses showing temperature differences of less than 0.5 $\mathrm{K}$. The detectors front end electronics is the warmest unit $(\sim 139 \mathrm{~K})$, due to its active dissipation, while the FPA operating temperature is only $0.5 \mathrm{~K}$ higher than its reference interface set at $95 \mathrm{~K}$.
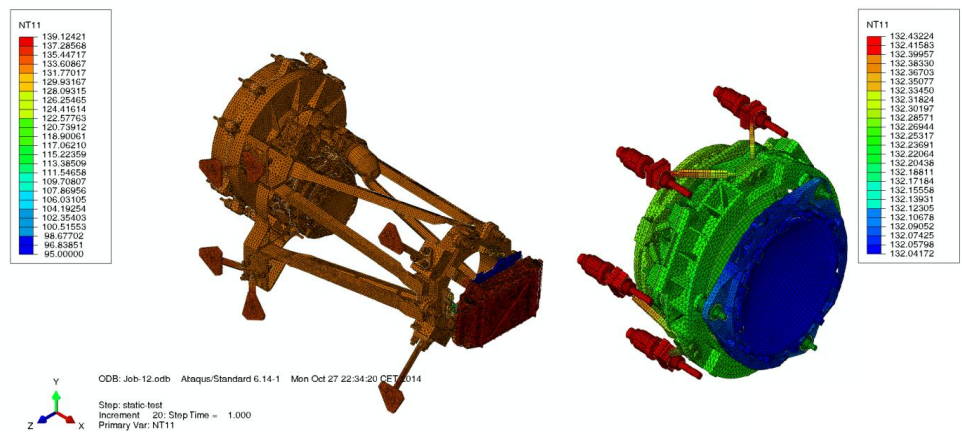

Figure 3-2. NISP FEM thermal model results: the NIOMA (left panel) and the CaLA unit with the lenses (right panel)

\section{The Optics (NI-OA):}

Main function of the NI-OA is the accommodation of the 4 lenses L1, L2, L3 and corrector lens L4. The first 3 lenses (L1- L3) are summarized in the Camera Lens Assembly (CaLA), while the single corrector lens is allocated in the Collimator Lens Assembly (CoLA).
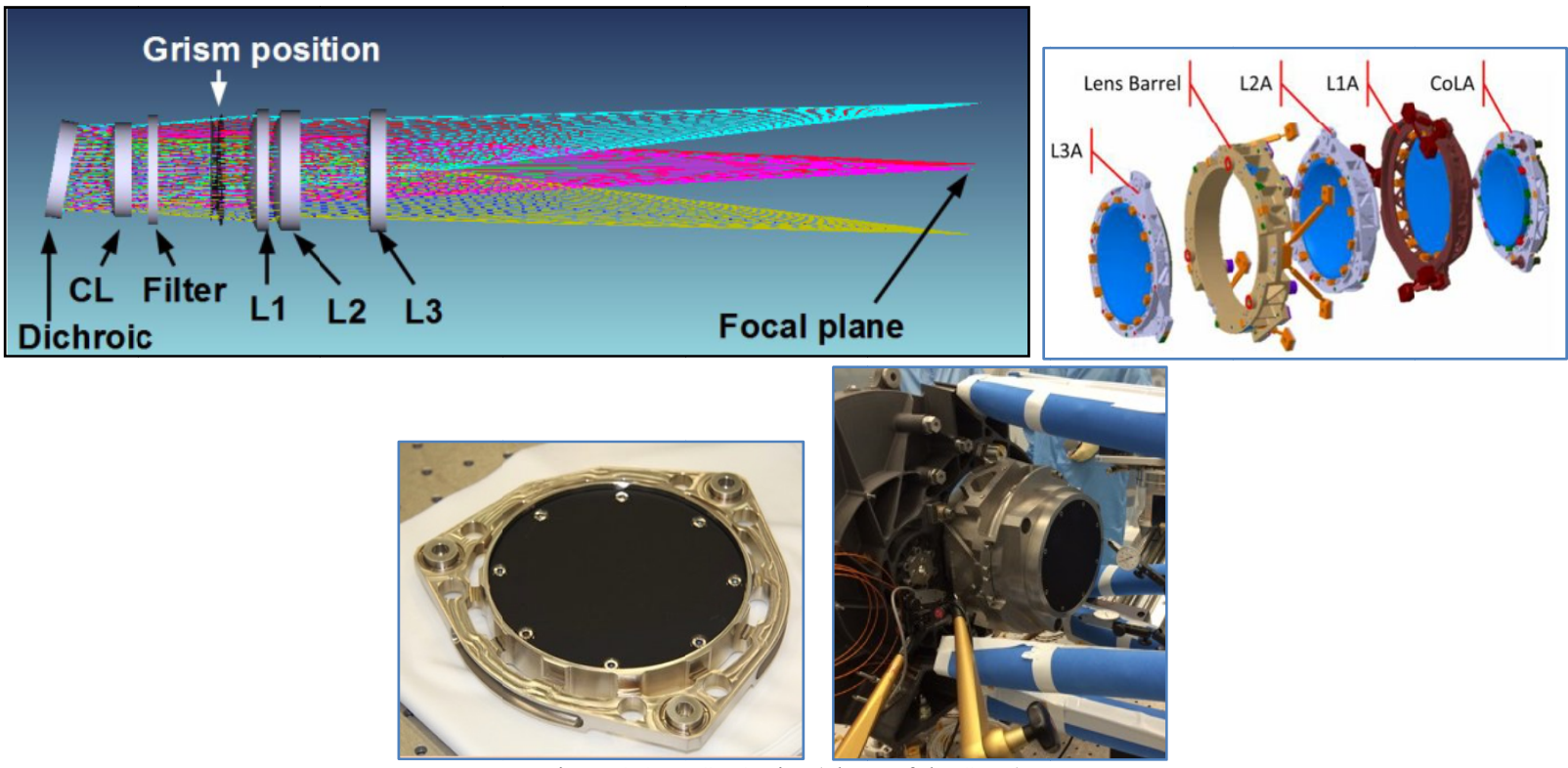

Figure 3-3: NISP Optics (photo of the STM)

The optical system operates in a temperature range between $132 \mathrm{~K}$ and $134 \mathrm{~K}$. Consequently, the lenses need to have different shapes during manufacturing, to reach the desired shape at operational temperature. For the "cold - warm" calculation, the warm lens geometry has to be derived from the ideal "cold" optical design, which is valid for one specific temperature of the operational temperature range. The transfer of the lens parameters from ops (operational conditions) to RT ("warm" room temperature) is dependent on the temperature dependency of material and optical parameters. 
At Room Temperature, the lens material constants are well known, however at the ops temperature range the most important parameters for the optical design, such as refractive index, CTE were only partially available for the used lens materials. The mechanical lens material properties such as CTE; strength, etc. are measured down to the operation temperature range.

The design drivers for the adaption rings are high precision, cryogenic operation temperature (110 K) and the large dimension of the lenses (168 mm). The design concept of the adaption ring is based on flexure hinges, which provide sufficient protection against vibration loads at ambient temperature as well as high precision $(< \pm 10 \mu \mathrm{m})$ and stability at cryogenic temperatures.

Criteria for the flexure hinge design are the low radial forces at cryogenic conditions to avoid any refractive index and polarization variations. The design is compliant with the large temperature differences between assembly and operation, the high precision and low-deformation requirements of the lenses, as well as with the deviating CTEs of the selected lens materials.

Each lens is glued (epoxy bond) in an adaption ring via a double pad, which provides the necessary elasticity caused by different CTEs of the lens and ring materials, as well as it allows a high position accuracy of the lenses relative to the lens barrel and the optical axis. The double pad itself is bonded to the lens using the same glue. The glue pad dimensions are the same for each spring with an accuracy $<0.1 \mathrm{~mm}$ respectively. Otherwise, asymmetric deformation of the corresponding lens is introduced after cooling down, and hence, the accurate position and form of the lens cannot be guaranteed. Also the high precision manufacturing process of the springs by means of wire eroding is of critical importance, since its thickness shows up with the third power in the spring force, which introduces additional lens movement at operational temperature of $\sim 135 \mathrm{~K}$.

Baseline for AR material selection is the similarity of the CTE of both lens and AR to minimize any stress in the lens material. The remaining force at OPS temperature is further reduced by the springs and so the lens deformation is kept as low as possible. Benefit of the design is that the assembled AR is mechanically very rigid and withstands the vibration loads at RT.

The assembled ARs of the CaLA lenses are mounted into the lens barrel, which provides high precision position of the lenses. The LB I/F the unit is decoupled from the SiC structure of by using 3 bipods.

The Filter wheel (NI-FWA):

The Filter Wheel Assembly (NI-FWA) is made of: A Cryo-mechanism, three filters, an "open" position for which the optical beam can cross the FWA without interference, a "close" position for which the optical beam is blocked, and one Wheel Structure in invar. The operational temperature is around $135 \mathrm{~K}$.

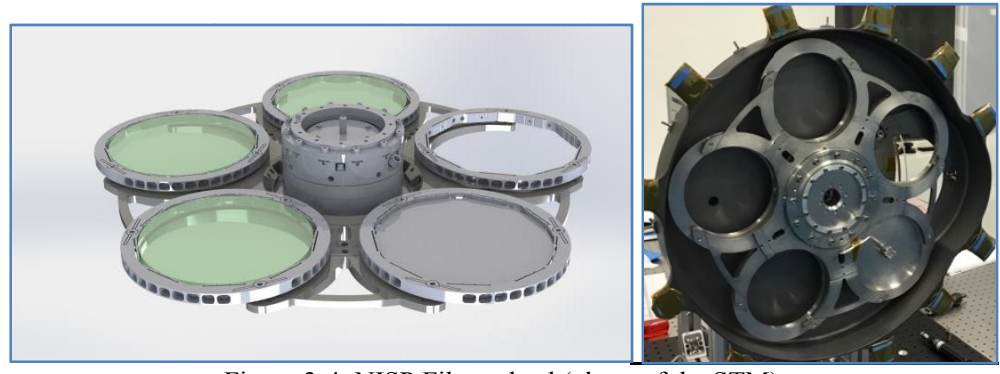

Figure 3-4: NISP Filter wheel (photo of the STM)

The Grism wheel (NI-GWA):

The Grism Wheel Assembly (NI-GWA) is made of: A Cryo-mechanism, four Grisms, an "open" position for which the optical beam can cross the GWA without interference, and one Wheel Structure in invar. The operational temperature is around $135 \mathrm{~K}$.
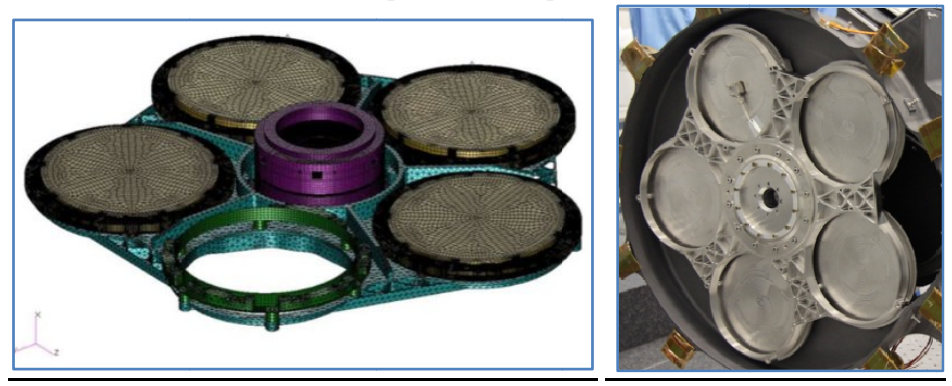

Figure 3-5: NISP Grism wheel (photo of the STM) 


\section{The Cryomechanism (NI-CM):}

The FWA and the GWA are motorized with two identical CryoMechanisms (CM). The CM operates from room temperature down to cryogenic temperatures $(120 \mathrm{~K})$. It includes a stepper motor that performs a coarse positioning, rotating the wheel at any of the 360 positions within $+/-0.3^{\circ}$ of uncertainty (due to the bearings frictions). When arrived at the required position, the motor detent torque (40mN.m) combined with the friction torques are enough to maintain the wheel position. During this motion, five degrees of freedom (DOF) are locked by the bearings assembly. The only DOF which is free is the rotation around bearings axis. The cryomechanism is powered only while actuation is required. When not operated, the cryomechanism is fully OFF.

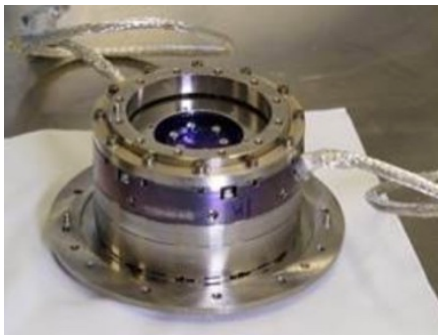

Figure 3-6: Cryomechanism breadboard model

\section{The Grisms (NI-GS, see ref [3]):}

Four Grism are mounted on the GWA, three "red" with a spectral bandpass [1250-1850 nm] and one "blue" with a spectral bandpass [920-1300 nm] The four grisms have about 14 grooves $/ \mathrm{mm}$.

Each NI-GS is composed of the grism itself (the optical element) glued on an invar mechanical mount. A baffle is mounted on the mount. The grism itself combines four optical functions in one component:

- A grism in Suprasil 3001 made of a grating engraved on the prism hypotenuse to make the light un-deviated at a chosen wavelength. In addition, a spectral wavefront correction is done by the curvature of the grating grooves,

- A spectral filter done by a multilayer filter deposited on the first surface of the prism,

- A focus function done by the curvature of the first surface of the prism.

The optical part of the grisms is glued in a mechanical Invar M93 ring through 9 flexible blades that compensate the small CTE difference between Suprasil 3001 and Invar. Three blades enable to minimize stresses in the optical element due to thermal differences (from $300 \mathrm{~K}$ to $130 \mathrm{~K}$ ) and to interface defaults.
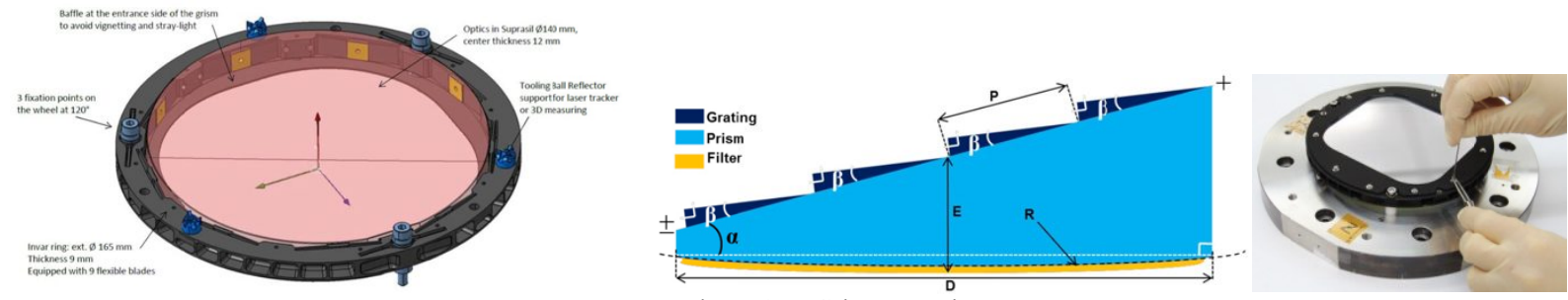

Figure 3-7: Grism overview

The Filters (NI-FI):

The three infrared filters (Y, J- and H-band) for the NISP instrument are realized as double-sided interference filters coated with the PARMS (plasma assisted reactive magnetron sputtering) process. Each side of the $\sim 12 \mathrm{~mm}$ thick Suprasil 3001 filter substrated is coated with a stack of up to 200 individual layers, resulting in a stack thickness up to $20 \mathrm{um}$ per side.

With a clear aperture of $126 \mathrm{~mm}$ (total diameter $130 \mathrm{~mm}$ ) this requires the use of coating machines designed for 8-inch substrates in order to achieve the required coating thickness homogeneity and to reduce the resulting transmissive wavefront error.

As the filter are not simple flats but rather lenses with $\sim 10 \mathrm{~m}$ focal length, manufacturing and verifying the uncoated substrates alone is complex enough, but furthermore the circumference (i.e. sides of the filter substrate) has to be polished down to $\sim 2 \mathrm{~nm}$ RMS roughness while retaining excellent circularity. This is necessary to allow controlled gluing of the filters into their mounts, later to be integrated into the filter wheel assemble (FWA). In previous studies we have also investigated the possibility to use the more conventional ion-assisted deposition (IAD) coating approach. While this process results in slightly better cosmetics of the filter surfaces, PARMS allows for better thickness homogeneity and was chosen for that reason. In the run-up to final filter production we have produced IAD prototypes of the H-band filter as well as a down-scaled PARMS prototype of the Y-band filter. Both test productions have resulted in well reproduced transmission properties, i.e. the transfer of the theoretical design with extended blocking and transmission $>95 \%$ in the passband worked very well.

After settling for the final filter bandpass design and coating method we have begun a new series of test coatings on smaller substrates for verification of the transfer from theoretical design to real filters. At the time of writing, the J-band samples have been evaluated, showing excellent transmission properties of more than $98 \%$ in the passband. Thermal shift of the bandpass between room temperature and operational temperature $(\sim 130 \mathrm{~K})$ has been reproducibly measured to be $<2 \mathrm{~nm}$ and well predictable. Measurements of the substrate bending due to internal coating stresses have been found to be in agreement with model predictions and within tolerance budget.

Ongoing work encompasses substrate manufacturing for qualification and flight models, test coatings of the $\mathrm{Y}$ and $\mathrm{H}$ filters and further qualification and evaluation of the coating properties. 

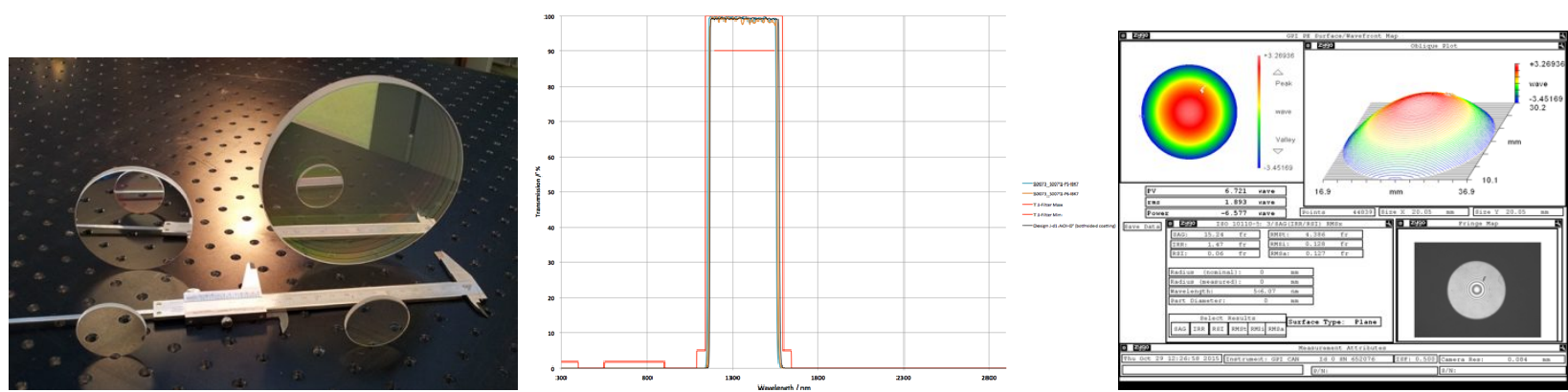

Figure 3-8: Y-band and H-band prototypes together with smaller witness samples / Transmission of the J band test coating on BK7 sample substrates.

Red lines indicate minimum / maximum requirements in the passband or blocking region, respectively. / Interferogram of a $20 \mathrm{~mm}$ diameter J band sample, coated on one side only. The focus term of $\sim 15$ fringes is within $15 \%$ of the theoretical prediction derived from Stoney's formula.

\section{Calibration Unit:}

The NISP Calibration Unit allows in-flight calibration of the infrared detector array. The unit provides stable illumination of the image plane at five different infrared wavelengths, allowing for small-scale flat field calibration and measurements of the detector linearity.

The design is relatively simple with $2 \times 5$ LEDs (one nominal and redundant per wavelength) inside the calibration unit pointing to a small patch of Spectralon PTFE material. The Lambertian scattered light is directly pointed towards the detector through a set of baffles without going through any of the optics.

Control of the LED brightness and thus received flux is performed by current and duty cycle regulation of the drive signal in the instrument control unit (ICU).

As the unit operates under cryogenic conditions, finding and qualifying suitable LEDs has become a major challenge, especially for longer wavelengths $>1.6 u m$.

Previous work has shown that commercially available off-the-shelf devices are not usable in our case. We have therefore procured the raw LED dies for the required wavelengths and submitted them to a uniform and controlled assembly and packaging process. The full formal space qualification of the LEDs is expected to take place well into the 2017. Nevertheless, initial tests of proofing devices, especially under cryogenic conditions, have confirmed that the LEDs are highly durable. The structural model of the calibration unit has passed vibration testing to confirm the validity of the mechanical design.

Current development steps include long-term cryogenic storage and lifetime cycle-testing of the LEDs as well as fine-tuning of the interior optical layout of the calibration unit.
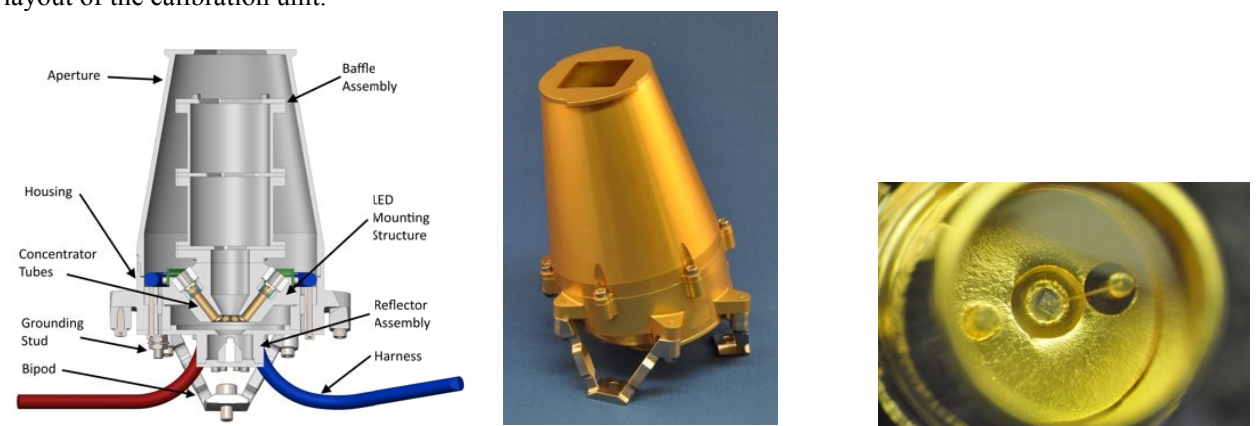

Figure 3-9: Cross-cut of the NISP calibration unit / View into one of the custom produced LEDs for the NISP calibration unit / The structural model of the NISP calibration unit, as used in the vibration campaign of the NISP STM. This model does not include LEDs, harness and optical elements.

\section{THE NISP DETECTOR SYSTEM (NI-DS)}

This assembly has the function to acquire the images by sampling the Field of View with an array of $4 \times 4$ IR sensors hybridized on multiplexers $(18 \mu \mathrm{m}$ pitch or 0.3 arcsec on the sky) and read out by the Sidecar ASICs. It is sequenced and read out by the NI-DPU processing to deliver digitalized data to the NI-DPU.

The NISP Detector System (NI-DS) is composed by:

1. A SiC panel called P4 (to be screwed directly on the SiC structure of the NI-OMA).

2. A Cold Plate (CSS) that supports the mosaic of $4 \times 4$ detectors. The Cold Plate is made of molybdenum and is held by three titanium bipods on the P4 Sic panel. A baffle (for detector protection), also made of molybdenum, is fixed on the CSS.

3. A support structure for the Sidecars (SSS). It is fixed onto the panel P4 by three bipods made of invar. The NI-SSS is made of aluminum.

4. The Sensor Chip System (SCS), composed of the H2RG sensor with $2.3 \mu \mathrm{m}$ cut-off (SCA), its cryo-flex cable $(10 \mathrm{~cm})$ and its ASIC sidecar electronic (SCE). 


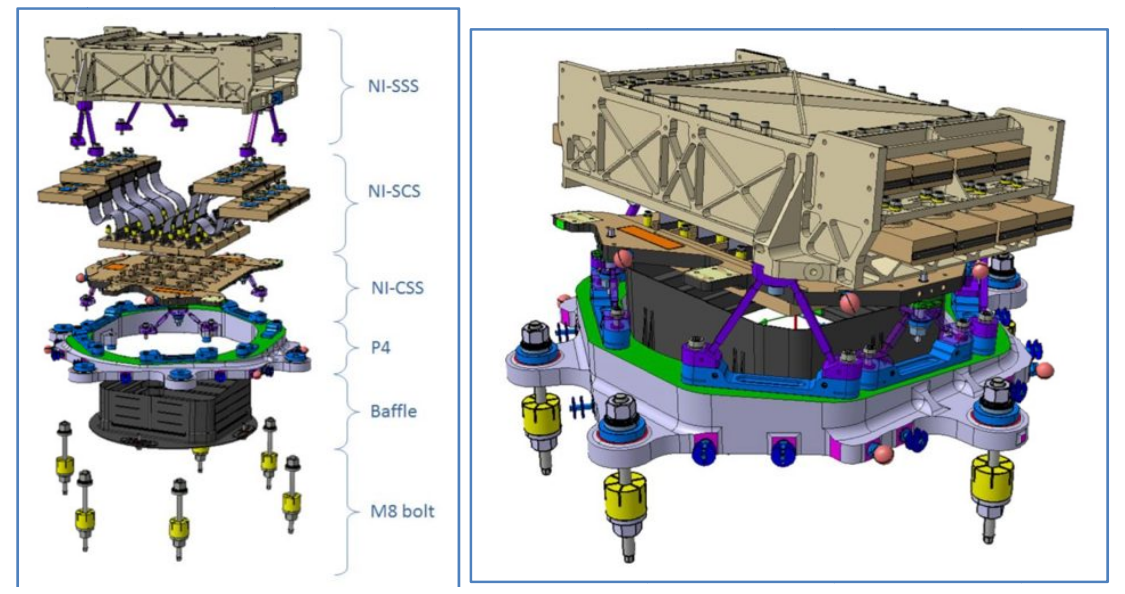

Figure 4-1: Focal plan overview

The operating temperature of the detectors (SCA) is lower than $100 \mathrm{~K}$ while each individual readout electronic (ASIC for digitization) operates at around $140 \mathrm{~K}$. Since the instrument units facing the detectors are controlled at a temperature below $135 \mathrm{~K}$, the resulting thermal emission up to $2.3 \mu \mathrm{m}$ ensures a very low thermal noise level. This configuration allows the optimization of the system thermal load on the satellite radiator and complies, at the same time, to all specifications in terms of noise.

SCA/SCE operation synchronism, at the level of a single master clock period (10 MHz, $50 \mathrm{nS}$ allocated maximum differential skew budget), is important for a mosaic made of tightly coupled detectors with potential electrical crosstalk. It is ensured partly by SCE firmware specifically developed for the NISP application (see next paragraph) and partly by specific HW in the DCU electronics. Basically, all the SCE systems are driven by a common master clock and all writings to the SCE internal registers (configurations and command directives) are synchronized by shift-registers clocked by the same master clock and started by a common pulse.

Data and power supply connections to each SCE are done by an unusually long double-shielded cable harness. The length is primarily dictated by internal distribution inside the payload module and the service module where warm electronics boxes are accommodated and by thermal decoupling reasons.

The kind of data communication (8 LVDS lines in parallel mode for the science data port, LVDS synchronous serial bidirectional lines for directives/housekeeping) and the requirements for the most critical power supply lines (SCE internal analog reference Vref, analogic supply VDDA and digital VDD2P5) have been already baselined and the hybrid harness is under study at Airbus DS. Several preliminary configurations have already been tested in order to evaluate critical aspects, such as LVDS master clock losses/duplication and critical power supply drops on the harness leading to specific circuits for the LVDS common-mode stabilization on the critical SCE clock line.

In particular, the power supply representative bread-board will mount the same DC-DC and continuous regulators, galvanic insulation system and ground/shielding concept as foreseen in flight with a representative $8 \mathrm{~m}$ length hybrid cable harness (PhBronze for the thermal gradient and $\mathrm{Cu}$ for the thermal stable sections). The SCA/SCE focal plane simulator (mounted in the same structure shown in the following figure) will be based on $4 \times$ SCA bare multiplexers driven by $4 \times$ NISP SCE operated at flight foreseen temperatures in a dedicated cryo-chamber. Final end-to-end performance tests for the DPU/DCU electronics will be carried out also inserting a fully operational SCE/SCA mounted on a liquid nitrogen vessel already operational at the OAPD laboratory.

SCE boot, configuration and data acquisition in the NISP standard mode (multi-accumulation) is possible both from by a Markury LTE controller, used as a reference system, and from the NISP data control units (DCU) demonstration models under test. Left side of Figure 4-2 shows the first DCU prototype during the first tests made to boot and drive a Teledyne ASIC.
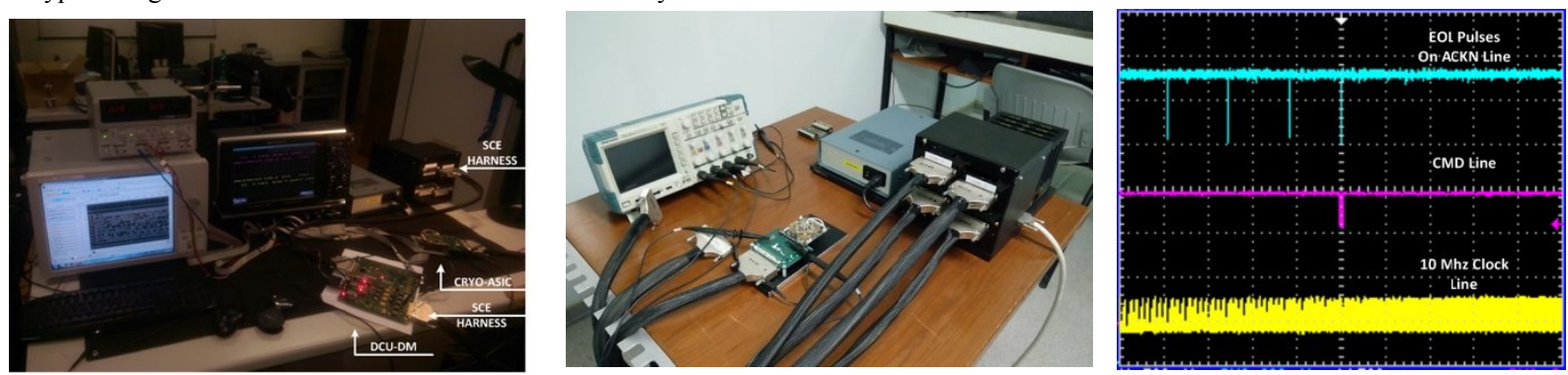

Figure 4-2 - Left side: DCU breadboard under comparative test against a Markury LTE controller. A Teledyne ASIC at room temperature is used as SCE reference. Center: The Markury controller handling 4x $8 \mathrm{~m}$ cable harnesses. Right side: SCE abort/Synchr directives reacting at EOL boundaries 
The Markury LTE system has been already refurbished and tested by the contractor (Markury, US) to support the NISP specific SCE microcode and also tested (See center of Figure 4-2) for proper operation with long cable harnesses and with the SCA mux/SCE operated at ambient and cryogenic temperature.

The specific SCE microcode (Electrical Engineering Firmware, EEF) has been previously delivered by Teledyne and is now under verification and refurbishment at Markury Scientific. Several upgrades are foreseen under interactive collaboration with Markury, the main enhancements to the EEF are:

- $\quad$ Possibility to generate end of line and frame pulses (EOL, EOF) on the SCE acknowledge return line. This feature is needed for debug tests and for inter-SCE synchronization verification

- Enhance the reactivity of the exposure Abort and Synchronize directives to react at end of line boundaries $(690 \mu \mathrm{S})$ instead of frame boundaries (1.42 S). This is essential to achieve synchronism of operation and precise exposure time stamp (See right side of Figure 4-2)

- Implementation of SCE/SKA internal Inter Pixel Capacitance test (IPC exposure) by means of simulated exposure on a selectable pixel grid

- Aliveness test. This has been implemented and is supported by an internal readable register incremented at each EOL, both during exposures and idle time

- Numerical UTR simulator. This is under implementation and allows to produce directly by the SCE simulated Multi Accumulation Ramps where each frame level is constant and the level increases with a programmable step

A large amount of detector characterization has been conducted with the engineering detectors and will be conducted for the flight detectors to obtain precise characteristics for noise, dark current, conversion gain, non linearity of the pixel response, QE, Inter pixel capacitance crosstalk, full-well capacity and persistence (latency) (ref [4] and [5]).

A demonstration model, with four detectors has been integrated and tested. Thermal Balance / Thermal Vacuum, conducted susceptibility at cold operational temperature, cold deformation measurement and vibration tests have been successfully done.
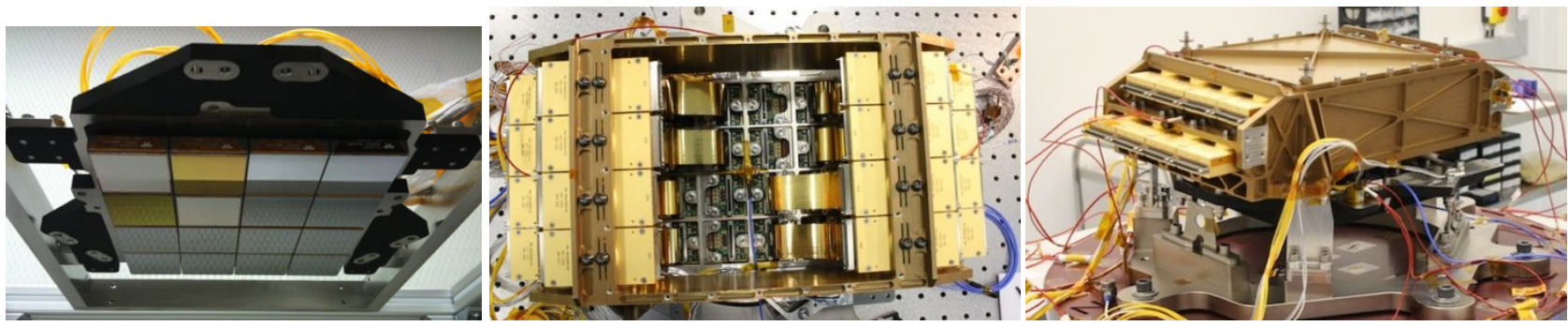

Figure 4-3: 4 Views of the NI-DS demonstration model

\section{THE NISP WARM ELECTRONIC}

The NISP warm electronic is composed of two Data Processing Unit (NI-DPU) and one Instrument Control Unit (NI-ICU).

\section{Data Processing Unit (NI-DPU):}

The full system is shown in the electrical drawing reported in figure "NISP functional electrical scheme", each DPU unit is mounted around a shared Compact PCI bus structure with the exception of the main power supply system and the $8 \mathrm{x}$ DCU boards each one managing one SCE/SCA detection pair. The two Data Processing Units (NI-DPU) are both including:

- $8 \mathrm{x}$ Detector Control Units (DCU) that provide clock and power to the readout electronic. In addition, these units will preprocess the data using FPGA boards

- Central Processor Unit that finalize the on board data processing, compress and format the data sending them via SpaceWire link to the central spacecraft memory

Each DPU is hosting the following boards:

CPCI Data Processors based with two Maxwell SCS750

CPCI Data Routers

CPCI Data Buffer

Power Supply

Except the DCUs all the boards in the DPU are cold redundant. Each DCU receives the data of one $2 \mathrm{~K} \times 2 \mathrm{~K}$ detector from one SIDECAR and performs the low level pre-processing foreseen in HW consisting of:

$\circ \quad$ Group of frames averaging

- Telemetry Extraction 
- Extraction of sub-sets of programmed raw detector lines to be used on ground for monitoring purposes

- Co-added Frame data buffering and Spacewire transmission to Data Buffer Boards

At this interface level redundancy is supported by the full duplication of DPU hardware. Averaged data groups can be configured to be transmitted to one of the Data Buffer Boards available in each DPU, this is accomplished by duplication of the $8 \mathrm{x}$ Spacewire (SpW) links. The same redundant configuration is available at each DCU TMTC interface bus: the control link based on the RS485 standard can be configured to be driven by one of the two available CPCI data router boards.

The Data Buffer board allows the storage of up to $46+46$ averaged frames with Telemetry and ancillary data from the $8 \mathrm{x}$ handled detection channels in double-buffering mode to ease the further data processing.

A functional demonstrator model of the DPU (with one Maxwell board, one DCU board and one Data Buffer board) has been manufactured and tested with a first version of the application SW.
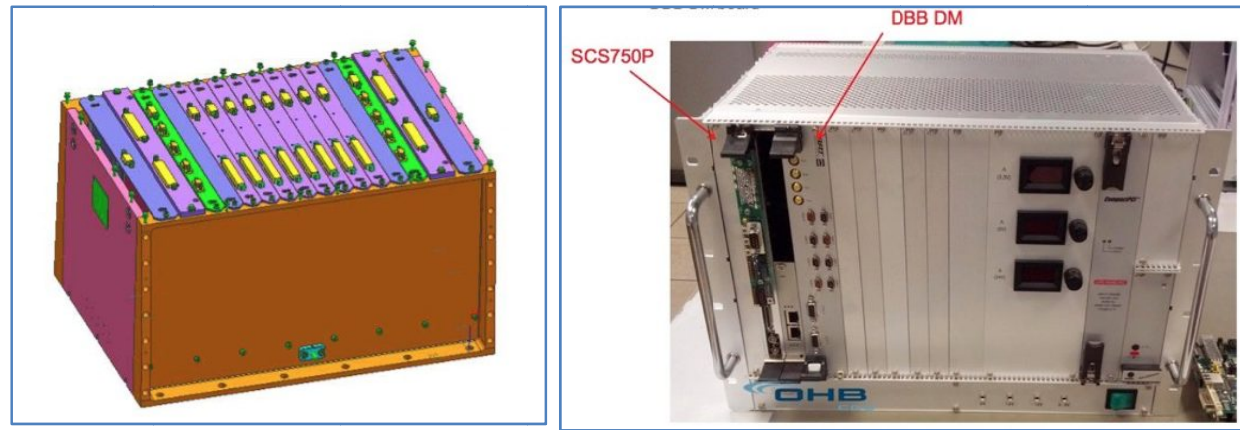

Figure 5-1: DPU (design / Demonstrator model)

\section{Instrument Control Unit Hardware (NI-ICU):}

- $\quad$ One Instrument Control Unit (NI-ICU) in charge of:

- Interface with the spacecraft via a 1553 bus for the commanding of the NISP

- Housekeeping management

- General power supply

- Command signal to the cryo-mechanism, to the 5 LED's calibration source and to the NI-OMA and NI-DS heaters (heater constant power is applied in open loop with power setpoint determined by ground operators)

The ICU has two sections (nominal and redundant), which are identical and operate in cold redundancy. Each NI-ICU (N or R) is divided in three boards, all of them interconnected by means of a backplane motherboard:

- $\quad$ LVPS (Low Voltage Power Supply): provides DC/DC converters to generate all the necessary secondary power supplies, as well as the 1553 transceivers for the NI-DPU link (1553 controller logic is actually located in the CPDU board).

- $\quad$ CDPU (Central Data Processing Unit): contains a LEON2-FT CPU embedded in a MDPA ASIC, which manages all the functions of the NIICU. This module also includes a RTAX FPGA that extends the functionalities of the MPDA, with the main aim of interfacing with the DAS module. The 1553 transceivers for the S/C link and the test connector are also located in this board.

- DAS (Data Acquisition System): this board features all the analogue acquisition and driving electronics that are used to interface with the rest of the NISP instrument, including the filter and grism wheels, heaters, temperature sensors and calibration LEDs.
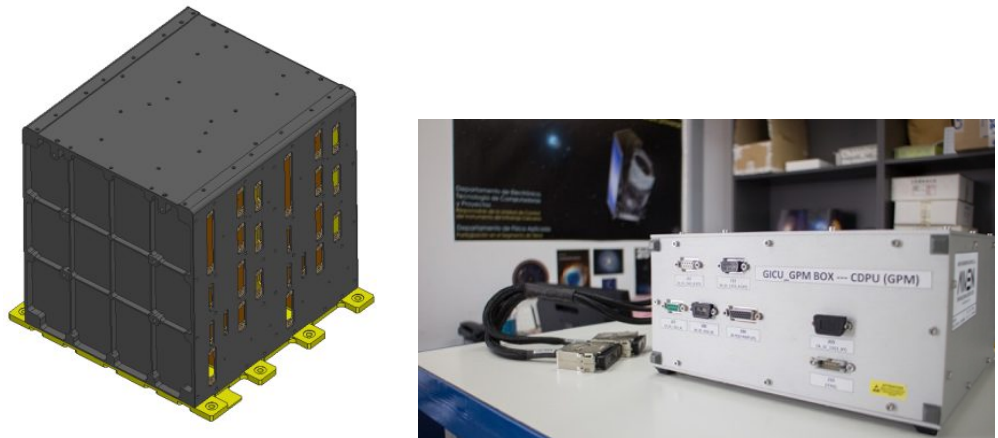

Figure 5-2: NI-ICU mechanical design (left) and EBB of the CDPU board (right). 


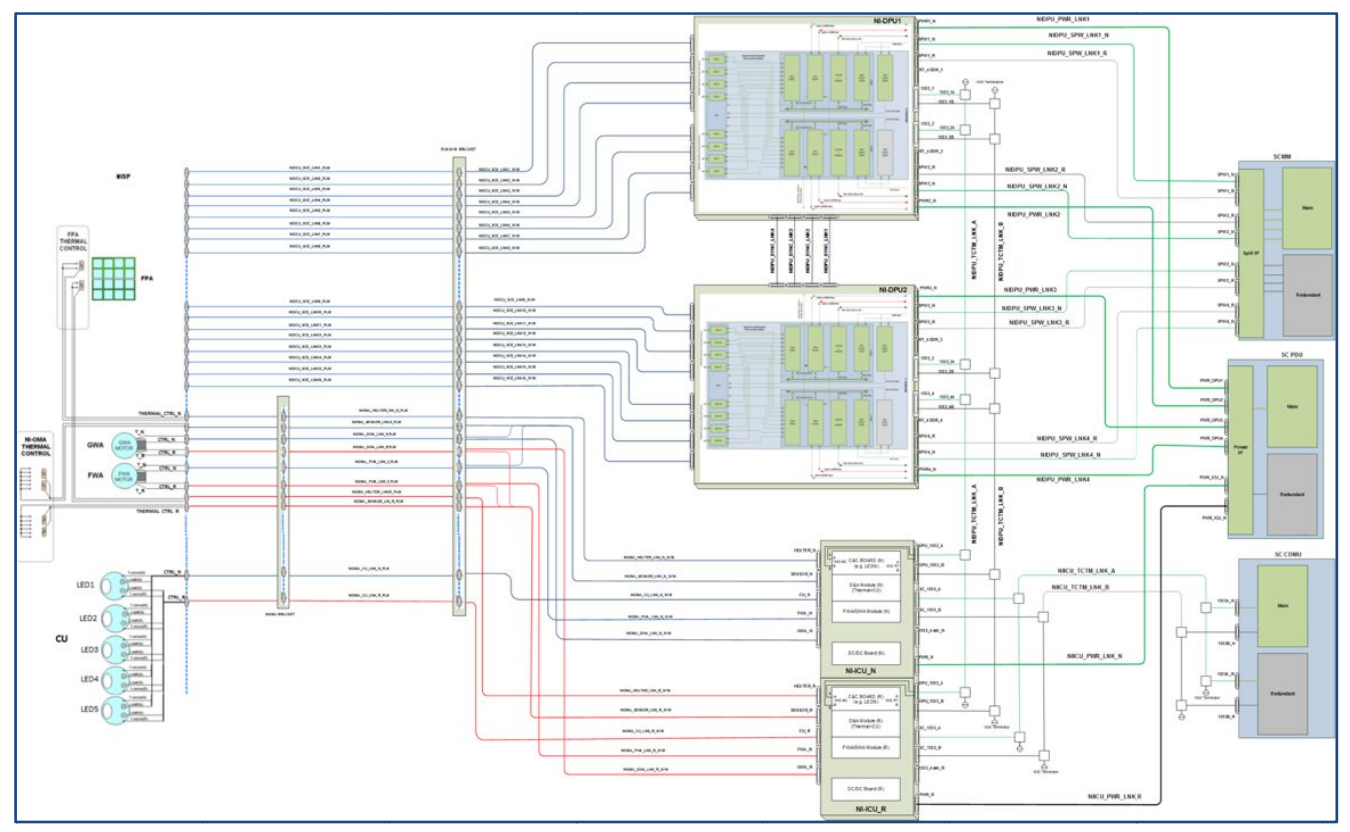

Figure 5-3: NISP functional electrical scheme

The warm electronic will be placed in the service module of the spacecraft at ambient temperature. A harness under Prime contractor responsibility provides the link with the NI-OMA and NI-DS. This cable will carry LVDS signal for scientific data, housekeeping signals, control command and power supply for equipment.

The main challenge of the warm electronics is to process the amount of data delivered by the detector during the integration of the following frame. The onboard data processing is complexified by the fact that the amount of downlink accepted to ground is very limited. Only final frames can be sent to ground, but as described later $\mathrm{HgCdTe}$ detectors deliver lots of frame to achieve final science performances.

The ICU Application SW (ASW) is devoted to manage the satellite/platform interface, the ICU/DPU interface and all the functionalities related to instrument commanding. It is in charge of the following functions:

- $\quad \mathrm{TM} / \mathrm{TC}$ exchange with S/C CDMU on Nom/Red 1553 link

- TC decoding and distribution to NISP instrument subunits: NI-FWA electronics, NI-GWA electronics, NI-CU electronics, NI-TC electronics, NI-DPU/DCU/SCE

- Global instrument monitoring and HK packet generation

- Time management, propagation of OBT to the DPUs, TM time tagging and high level instrument internal synchronizations

- $\quad$ NISP operating mode management

- Execution of autonomous functions and FDIR algorithms and processes.

- Control of the calibration unit (ON/OFF, intensity level and current absorption handling)

- Control of filter wheels (reference position, position switch)

- Thermal control (open loop) of the NI-FPA detector cold-plate through temperature sensors and heaters

- High level handling of macro-commands submission to detector system

- Thermal control of the NI-OMA through temperature sensors and heaters

- $\quad$ Management of software maintenance, memory patch and dump (EEPROM patching is performed by the Boot SW)

Instrument Control Unit Hardware Application Software (ICU ASW, see ref [7]):

The ICU ASW is based on RTEMS real-time operative system, in the space-qualified version by EDISOFT.

Telecommand and Telemetry packets will be based on the Packet Utilization Services (PUS) standard, with the implementation of services tailored to the specific needs of the Euclid project.

A coordinated effort is in place with the Prime of the Spacecraft and with the VIS CDPU ASW team in order to ensure a common approach and, as far as possible, implementation of services between NISP and VIS, so that the SW interfaces with the Spacecraft can be simplified and standardized. The interface with the DPU is based on a second MIL-STD-1553 bus, similar to the one used between the Spacecraft and the Euclid instruments, in which the DPUs are configured as Remote Terminals and the ICU as the Bus Controller. The SW interface and communication protocol is an internally defined one, with the aim of reducing as much as possible the load of management tasks on the DPU processor, since this resource is needed for the demanding data processing tasks. The ICU ASW will decode the PUS formatted high level TCs and implement the low level sequences towards the two active DPUs. 


\section{ONBOARD DATA PROCESSING (SEE REF [8]):}

The routine science NISP operations foresee 20 fields of observation per day, each one composed by four dithers where four exposures each are taken, for a total maximum assigned science data telemetry of $290 \mathrm{Gbit} /$ day. A dark exposure is taken during the spacecraft slew. This limited amount of allowed telemetry, together with the huge number of frames typically produced by IR detectors operated in multi-accumulation mode, have as a consequence the need to perform part of the processing pipeline directly on-board and to transfer to ground only the final products for each exposure. Moreover, final data must be also compressed to fit with the assigned telemetry throughput. A number of readout modes have been envisioned for the NISP instrument in the various development stages. Multi-accumulation (MACC) is at the moment the preferred modes for both spectrograph and photometer readout. MACC readout is a peculiar Up the Ramp process (UTR) where detector readouts are grouped in contiguous sets of readouts uniformly placed along the accumulated charge ramp. The data processing can be split into two main stages: stage 1 is implemented in the NI-DCU, directly interfaced to the SCS, where the first static basic pre-processing steps are performed, while stage 2, performed in NI-DPU, is devoted to the processing and compression of the final data frames.

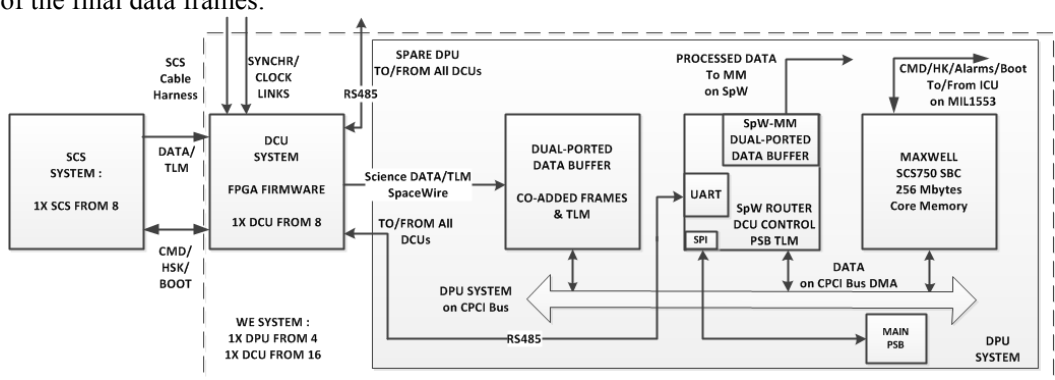

Figure 6-1: Pre-processing HW structure connected to $1 \times$ SCS single pair $($ H2RG SCA + SCE) from a total of $16 \times$ located inside the SCS system

The software architecture is dictated by the science requirements and depends on the hardware organization, in terms of DPU power, internal memory, available links with both DCU and SVM. During the previous different phases of the project various processing possibilities were analyzed, in terms of computational complexity, DPU internal memory needs, amount of final data and quality of results. As a result, the foreseen on-board pre-processing pipeline $^{7}$ will be as depicted in Figure 6-2 where the violet blocks represent the operations performed inside the DPUs. This operational flow is sequentially repeated to cover the 17 exposures ( 4 spectro +12 photo +1 dark) to be performed during each single cycle.

At the end of the pipeline described in Figure 6-2 final generated data, with their associated header and metadata to properly re-construct images on ground, are transmitted to the spacecraft Mass Memory Unit, to be down-linked to ground.

The most crucial constraint for the on-board processing is given by the need to keep up with the on-going observations, so the previous work was mostly concentrated to verify the algorithm performances, especially in terms of time spent. Current development steps include the integration of the data processing with the overall DPU Application Software structure.

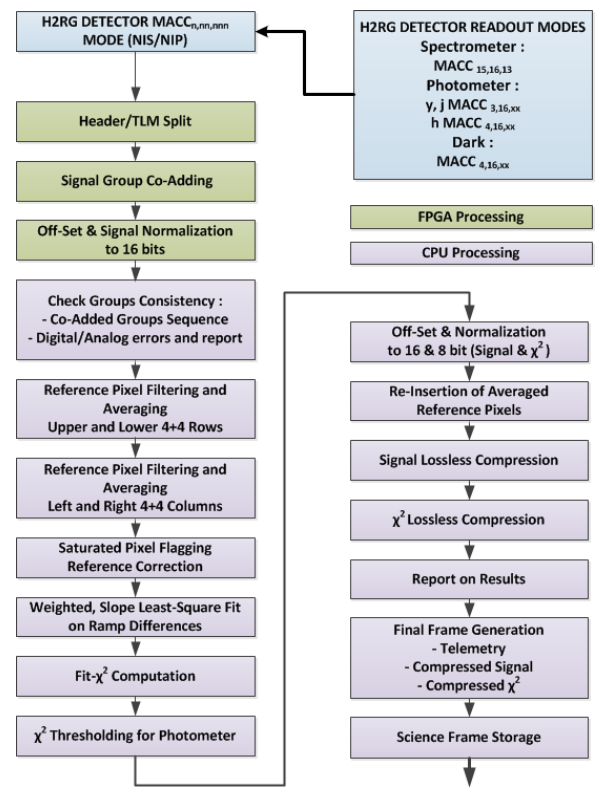

Figure 6-2: On-board data processing pipe-line for the Euclid NIS/NIP instrumental modes. The pipe-line is subdivided in three different sections on the base of the involved hardware, in the order: SCE analog hardware, FPGA hardware and sequential processing hardware 


\section{NISP PERFORMANCES}

Optical performances

The following figure shows the evaluation of the Encircled Energy compared to required values (the maximum radius of the Encircled Energy (EE) at $80 \%$ and at $50 \%$ shall be lower than the specified ones. The following figure shows that NISP complies with its optical requirement.

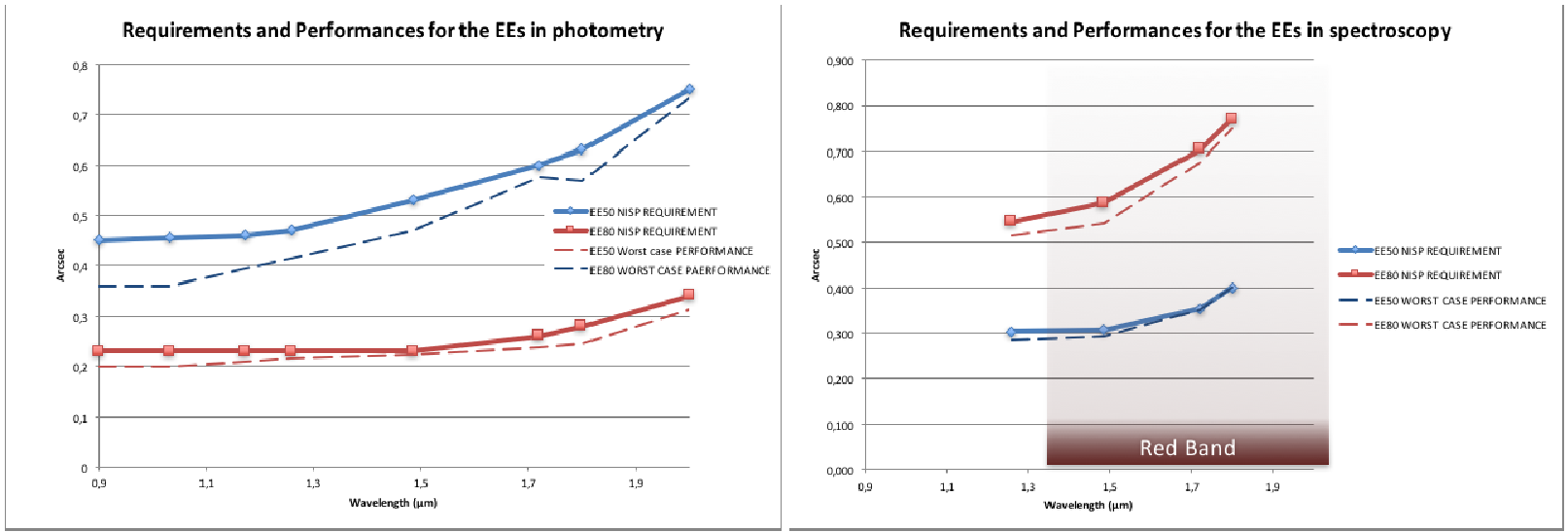

Figure 7-1: EE (Encircled Energy) performance evaluation

\section{$\underline{\text { IR detector QE and noise performances: }}$}

The detector QE and the detector noise are a major concern for the future NISP performance. One important goal is to ensure not only mean specified values but also that $95 \%$ of pixels meet these requirements to ensure the efficiency of coverage in the full survey.

With some detectors already produced by Teledyne Imaging Sensors under NASA contract, the first measurements have shown excellent flat field response and Quantum Efficiency within the $(0.92-2.3)$ band. The following Figures show results from 18xxx detectors corresponding to flight parts recently produced and $17 \mathrm{xxx}$ detectors corresponding to the ESA NRE phase production of 2.3 um cutoff H2RGs.
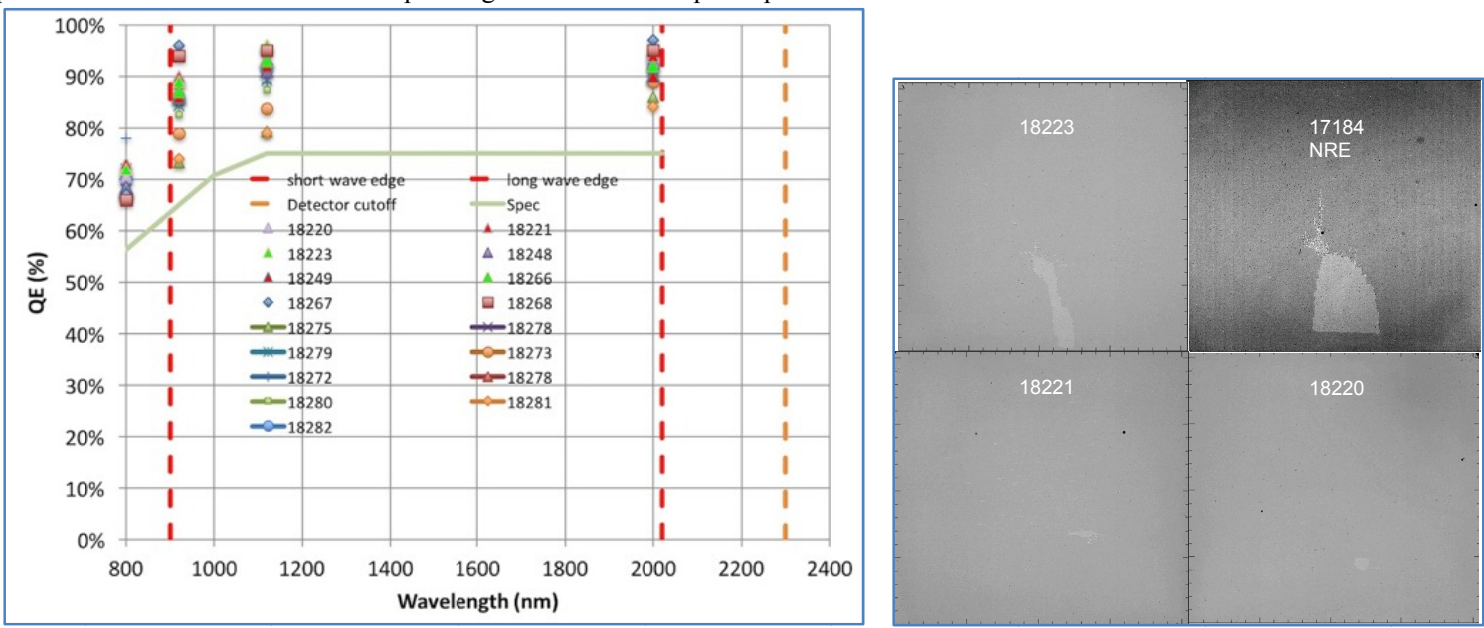

Figure 7-2: QE measurements on the first flight detectors between 920nm and $2300 \mathrm{~nm}$ (left). Flat Field images of flight detectors compared with NRE phase detectors produced shows an improvement in the spatial homogeneity of the pixel responses.

The homogeneity of the pixel response is excellent. The CDS readout noise shows the same performances demonstrated during the NRE phase. The mean dark has been shown to be very low around $0,005 \mathrm{e}^{-} / \mathrm{pix} / \mathrm{s}$ at $100 \mathrm{~K}$ with a sharp distribution and well inside NISP specifications.

From these first tests on Flight parts, we can expect a very high quality NIR Focal Plan. 

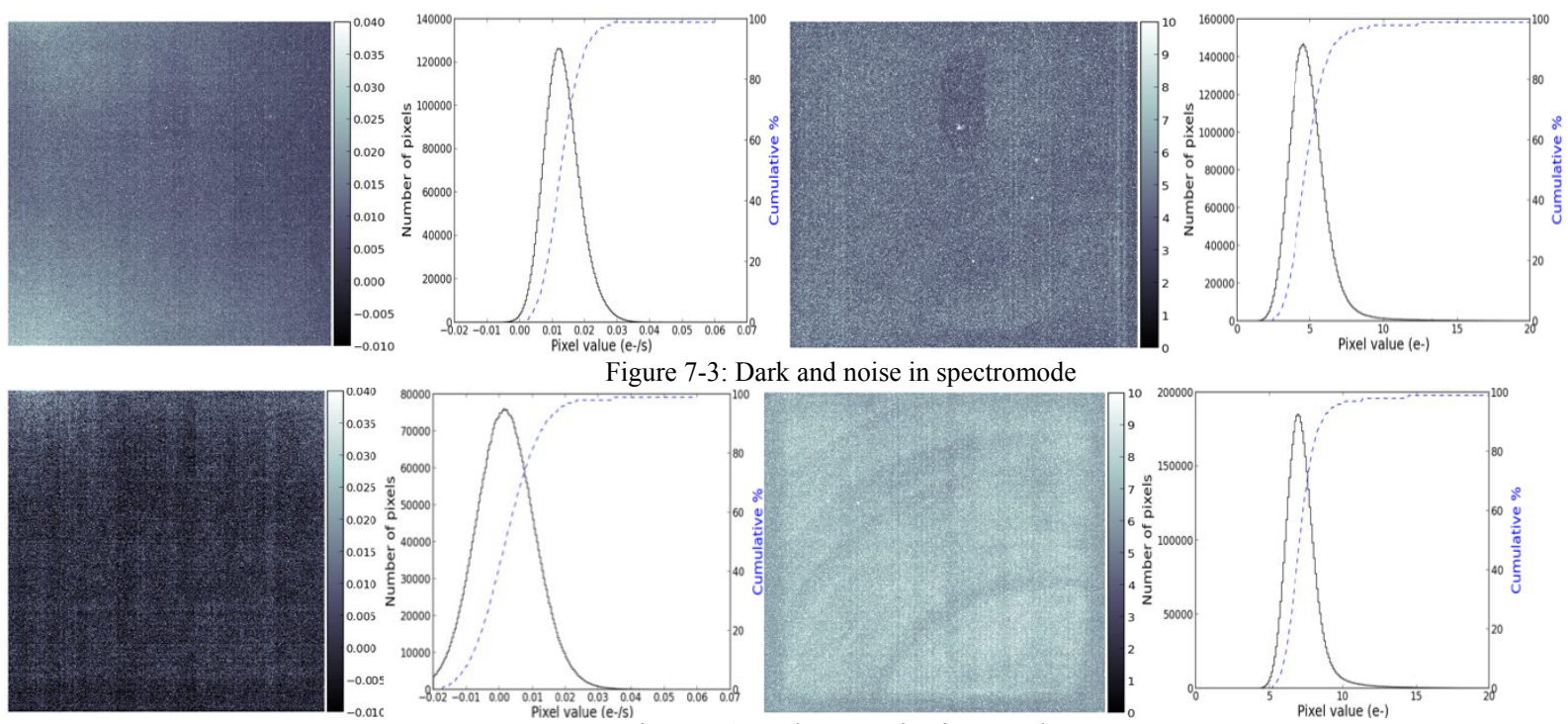

Figure 7-4: Dark current in photo mode

\section{DARK and NOISE during DM tests}
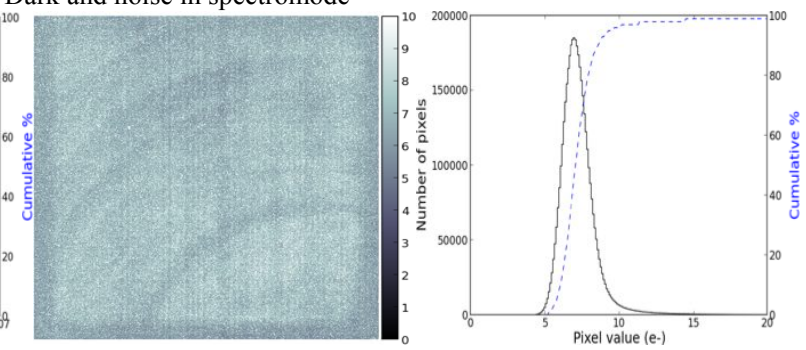

The tables below summarize the properties of both Asics under study. Apart from the photometry noise, which is a bit higher than the science requirements (due to defectice grounding scheme during the tests), all other values are very good and comply with the science requirements. As may be expected, noise (whether in spectrometry or photometry mode) is not very sensitive to temperature variations, while dark current clearly increases with temperature. This is well observed with Asic4.

\begin{tabular}{|c|c|c|c|c|c|c|}
\hline SCA17191 & & TIS report & Median & Error & $95 \%$ & Requirement \\
\hline CDS(e-) & & 10.77 & 16.87 & 0.24 & 20.87 & $\mathrm{n} / \mathrm{a}$ \\
\hline Macc photo (e-) & & & 7.52 & 1.00 & 10.01 & $<9.0$ \\
\hline \multirow[t]{2}{*}{ Macc spectro (e-) } & & & 6.90 & 2.05 & 10.69 & $<13.0$ \\
\hline & $80 \mathrm{~K}$ & & 0.0145 & 0.0046 & 0.025 & \multirow{3}{*}{$<0.07$} \\
\hline \multirow[t]{2}{*}{ Dark current (e-/s) } & $90 \mathrm{~K}$ & & 0.013 & 0.0035 & 0.023 & \\
\hline & $100 \mathrm{~K}$ & 0.009 & 0.018 & 0.0037 & 0.032 & \\
\hline SCA17245 & & TIS report & Median & Error & $95 \%$ & Requirement \\
\hline $\operatorname{cDS}(\mathrm{e}-)$ & & 12.56 & 16.68 & 0.23 & 20.25 & $\mathrm{n} / \mathrm{a}$ \\
\hline Macc photo (e-) & & & 7.21 & 0.96 & 9.60 & $<9.0$ \\
\hline \multirow[t]{2}{*}{ Macc spectro (e-) } & & & 6.78 & 1.90 & 10.03 & $<13.0$ \\
\hline & $80 \mathrm{~K}$ & & 0.0008 & 0.0059 & 0.013 & \multirow{3}{*}{$<0.07$} \\
\hline \multirow[t]{2}{*}{ Dark current (e-/s) } & $90 \mathrm{~K}$ & & 0.0033 & 0.0036 & 0.013 & \\
\hline & $100 \mathrm{~K}$ & 0.033 & 0.0077 & 0.0032 & 0.021 & \\
\hline
\end{tabular}

Figure 7-5: Dark and noise results

Conducted Susceptibility:

Conducted susceptibility of Euclid SCS in nominal conditions has been measured in differential and common mode by CDS noise comparison with and without perturbation injection on bias lines between RO electronics and SCE (see [8]). This sensitivity appears to be quite low excepted in the 5MHz$100 \mathrm{MHz}$ band and pixel reference correction is very efficient to prevent its noise to be injected on science data

In a non-surprising way, (given the specs asked by Teledyne ) the sensitivity of VDDA and Vref is higher than for other biases.

Figure below represents the readout noise (in ADU), without reference pixel correction, for a $74 \mathrm{~dB} \mu \mathrm{V}$ injection on VDDA line. Red line is the noise floor with 0 injection. The large increase between $10 \mathrm{MHz}$ and $100 \mathrm{MHz}$ is difficult to see when reference pixel correction is "on"

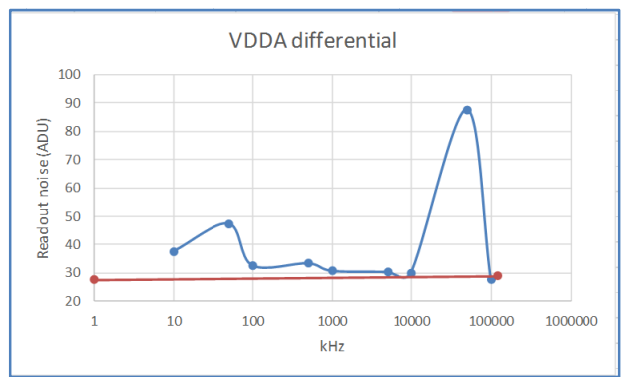

Figure 7-6: Conducted susceptibility measurement 


\section{Global performances:}

The performances for both channels have been explored in more detail during this year. The current radiometric budget has been verified with an updated evaluation of the optical throughput information of each element and is given in the following figure.
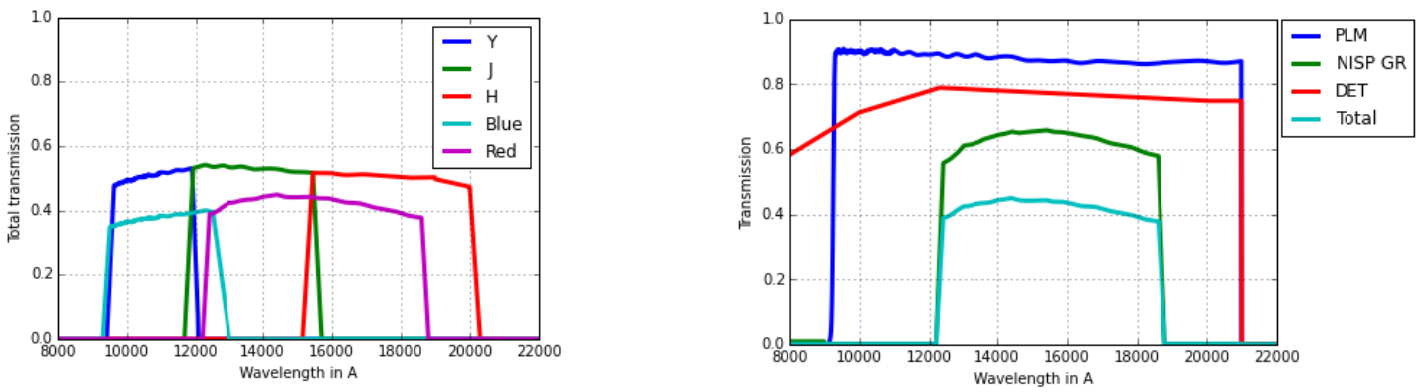

Figure 7-7: Best estimate of the PCE for photometry on the left and spectroscopy on the right

A NISP optical model has been built with all optical elements described above using Zemax and has been used to perform an evaluation of optical performance of NISP at $95 \%$ (encircled energy, PSFs). It has shown to be well inside specifications. This optical model is used also to derive a library of PSFs for both chanel to verify final performances. The ground campaign on the flight model will be set to verify and adjust the precision of these models on the final instrument and will then validate the modeling for further calibration on flight.

To verify the full NISP performance and models, tests will be done on ground directly on the flight model starting end 2017 (see details in next section). Many prior tests will be done first at sub system level next year. Then, prior to the final integration, an electrical model will be built mid 2017, and will allow to test the warm electronic and the focal plan with 4 detectors. This will give a first global verification of the full detector chain performance. A full performance campaign will be done then on the flight model and will allow to verify first the instrument functionalities but also to perform measurements needed to prepare flight calibration, as for example PSF measurements (both photometric and spectroscopic) or a full characterization and validation of the spectroscopic wavelength solution of the grism.

To meet the science requirements, the imaging mode of the NISP instrument is required to have a depth of $\mathrm{YAB}, \mathrm{JAB}$ and $\mathrm{HAB}=24 \mathrm{mag}(5 \sigma)$ for a point source with a high image quality defined in terms of radii of encircled energy (EE50, EE80) of $(<0.30,<0.62)$ arcsec, $(<0.30,<0.63)$ arcsec and $(<0.33,<0.70)$ arcsec at the center of the $\mathrm{Y}, \mathrm{J}$ and $\mathrm{H}$ bands respectively. The NISP photometry channel has progressed with a slight change in filter band passes. The blue cutoff of the Y-bandpass was before set to be at $920 \mathrm{~nm}$, given by a fast transition of the dichroic splitting the light by wavelength and reflection into VIS and transmission into NISP. However, the switch between these two modes was not as fast as originally hoped so that at $920 \mathrm{~nm}$ still substantial light would not yet be passed into NISP. Together with the finite edge width of the Y-filter in NISP this would have created the uncomfortable situation of the Y-bandpass being defined by a superposition of dichroic and filter edge, potentially varying over the field-of-view. To regain control, it was decided to shift this edge by some $30 \mathrm{~nm}$ to the red and only start the complete in-band at around $965 \mathrm{~nm}$. The other filter edges remain unchanged, although to optimize the filter manufacturing the flight filters might show very steep edges but become slightly wider, so that they slightly overlap. In any case the filter band passes will be finely measured in the lab before flight.

An SNR evaluation has been down for the mission PDR to verify the sensitivity of the photometric channel using the full sky noise evaluation. One example of map is shown on Figure 78 This analysis allows verifying that the SNR requirement ( $\mathrm{SNR}>5$ on a $\mathrm{mAB}=24$ point source object) is reached with margins on average over the reference survey (mean $\mathrm{SNR}=5.8$ ).

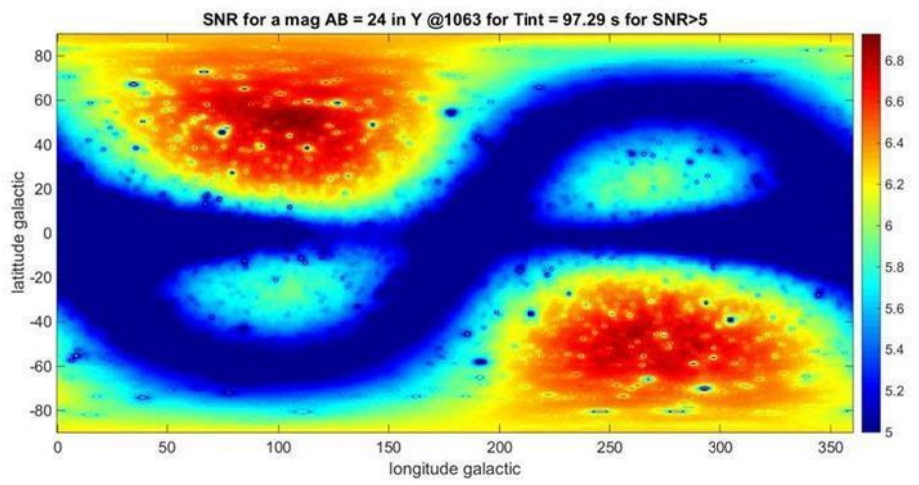

Figure 7-8: SNR map (galactic coordinate) Current Best Estimate of the System in NISP P Y band channel. SNR reached after 3 exposures (out of 4) on $\mathrm{mAB}=24$ object in $3 \times 3$ aperture.

In spectroscopy, the sensitivity is based on the detection of the $\mathrm{H \alpha}$ line in galaxies spectra in the range of redshift of $0.7<\mathrm{z}<1.8$ and is computed to estimate the compliance with the requested limit flux of $2 \times 10-16 \mathrm{erg} \mathrm{cm}-2 \mathrm{~s}-1$ at $>3.5 ?$ for all objects over the entire wavelength range.

The SNR has been estimated for the $\mathrm{H} \alpha$ line and is shown on the following figure. 


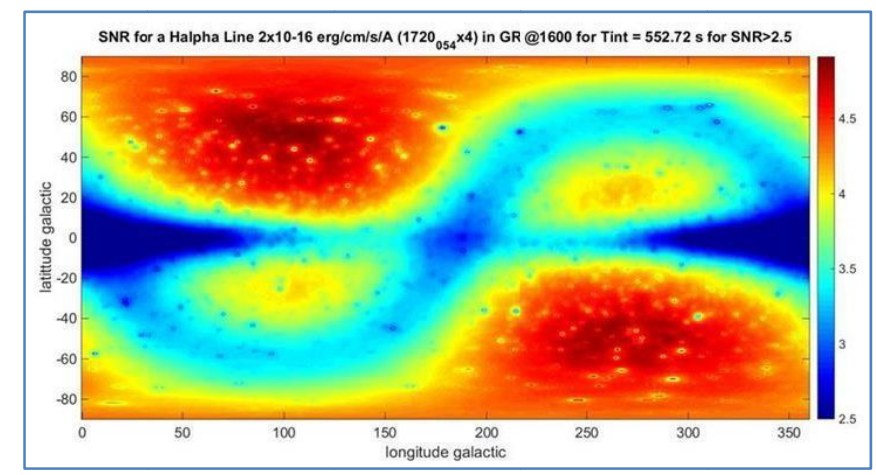

Figure 7-9: SNR map (galactic coordinate) Current Best Estimate of the System in NISP red grism channel. SNR after 4 exposures on line of flux of $2 \times 10-16$ erg cm-2 s-1@1720 nm in 4x4 aperture.

To verify up to science level, and to take into account the impact of contamination of objects in the field, an advanced end-to-end simulation pipeline for spectroscopy is used (Garilli et al. 2014) using simulated images from an Euclid NISP simulator (Zoubian et al. 2014), with a realistic input galaxy distribution to evaluate the expected completeness and purity of the resulting selected spectroscopic sample.

For the mission PDR, we have studied the dependence of purity and completeness on the different noise sources: zodiacal background, in and out of field stray light from the telescope, cosmic ray hits and persistence coming from the H2Rg detectors, using the most advanced models provided by NISP and the project for telescope stray light (ref [1]) and detector persistence (Serra et al. SPIE 2015). We have shown that zodiacal light and Out-ofField stray light are the main sources of sensitivity degradation is spectroscopy, the other effects contributing for about $5 \%$ globally on purity and completeness. As the in-field stray light and persistence effects are very difficult to be taken into account in the survey, we have explored in detail the effects of only zodiacal light, Out-of-Field stray light and stellar density on the full survey through an E2E simulation chain, applying the other effects posteriori on a purely statistical basis. We have simulated observations for 12 'reference scenarios' (each covering 1 square degree), spanning different conditions of environmental conditions, and computed the completeness and purity through the full chain of image simulation, spectral extraction, redshift measurement and redshift validation.

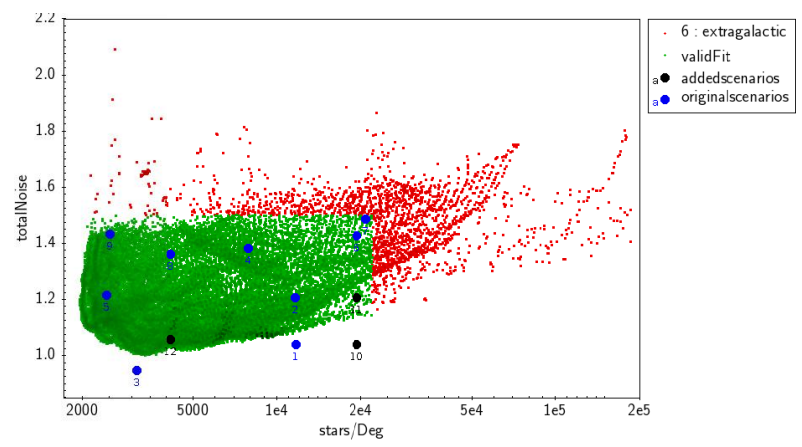

Figure 7-10: Total noise in spectroscopy in function of star density for typical pointing in Euclid in green. The noise in each simulated pointings are indicated by blue points

The completeness and purity obtained depend on the total noise, defined as the quadratic combination of noises due to the zodiacal light and the Out-ofField stray light, as well as on the redshift and on the line flux. Hence, for each redshift and line flux bin, we have fitted the simulated reference grids of values estimated for the success rate (or completeness) and purity as a function of the two variables total noise and stellar density, with corresponding smooth surfaces interpolating over the whole survey. This allowed us to obtain a value for the success rate, completeness and purity for each of the pointings of the reference survey. Posteriori, we further applied a mean decrease of 5\% in purity and completeness to account (at least statistically) for the effects of persistency, in-Field stray light and cosmic ray hits.

By including the stray light and after correcting from purity, only scenarios with total noise lower than $1.2 \mathrm{e} / \mathrm{s}$ are compliant with the requirement of galaxy density with good redshift for the survey $\left(1700 \mathrm{gal} / \mathrm{deg}^{2}\right)$. Beyond the NISP spectroscopy performance, we illustrate here the nuisance of stray light from star and star density on the high-level performance of the Galaxy Clustering science, as expressed by the top level requirement.

This example is only indicative as several improvements on the data processing and the sky model are not taken into account at this stage but it was done to give indication that scientific performance of Galaxy Clustering cannot be assessed without including the fully optimized NISP data processing chain, the complete masking procedure, an optimized field selection and field weighting scheme, including a severe rejection of dense stellar fields from the Euclid survey. This work will be extended now at mission level to optimize the survey for both weak lensing and clustering side. 


\section{NISP MODELS AND DEVELOPMENT}

Three different models to be developed are the following:

A DM (Demonstration Model) / STM (Structural and Thermal Model):

- $\quad$ To validate the design of the NI-OMA \& NI-DS structure and thermal control by doing the vibration and the TB/TV tests

- To check, at NI-DS level, the EMC susceptibility of the NI-SCS (detector/flex cable/sidecars) and to prove the auto compatibility of four SCS working together and synchronized.

This model has been integrated and successfully tested (see in the NI-DS section for the NI-DS DM tests).

During vibration, the measured first frequency is very close to the prediction (higher by $2 \mathrm{~Hz}$ )

The amplification measured in sine and random for the structure and for the different subsystem are generally (not always) higher than expected, then deeper notching than expected has been requested and applied. The accelerations on the different parts of the STM have seen quite reasonable levels both in sine (roughly around $13 \mathrm{~g}$ for any axis) and for random (roughly between 5 grms and $10 \mathrm{grrm}$ ).

The interface forces have been measured. The requested interface forces have been obtained.

During TB/TV, as first quick analysis show that the STM model is behavior is as expected, particularly, the gradients in the structure and between the different components are as expected. The impact of the transient due to the FWA and GWA activation is as expected very low.

This model will be delivered to ESA as STM in July 2016.
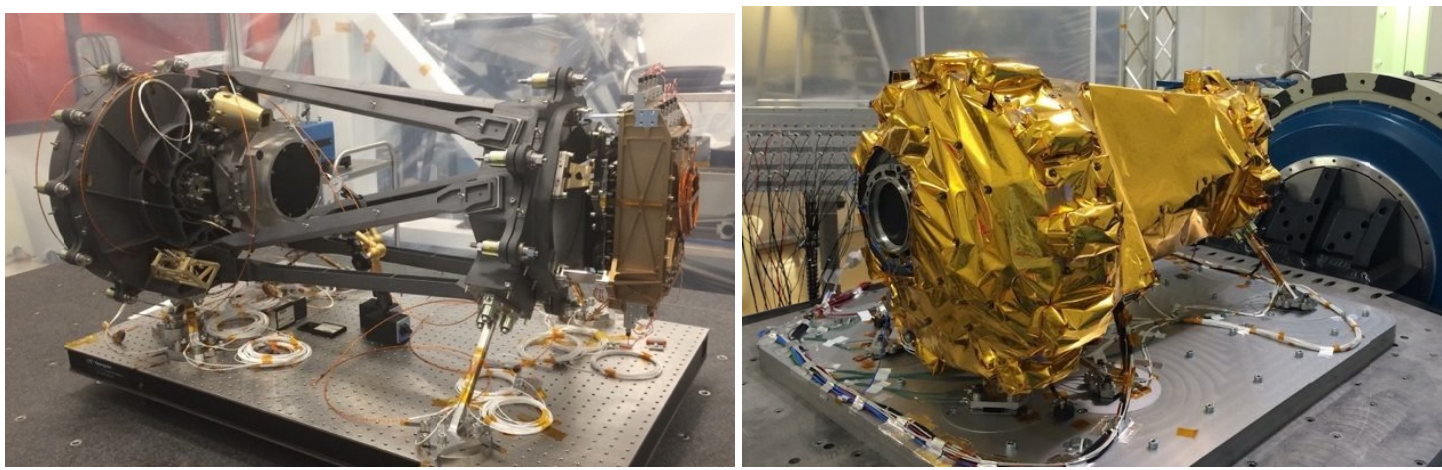

Figure 8-1: NISP STM model

An Engineering Model (EM) / Avionic model (AVM):

An engineering model of NISP will be developed. It will consist of all the NISP subsystems qualification model (QM) excepted the structure and the optics. The NI-DS EQM will have a four engineering NI-SCS. Flight representative harness will interconnect the NI-ICU, NI-DPU, NI-DS, NI-TC, NIFWA, NI-GWA and NI-CU as for flight. The EM will be tested under vacuum at cold operational temperature (135K for CU, GWA and FWA; [85K$100 \mathrm{~K}]$ for the detector and $140 \mathrm{~K}$ for the sidecar electronics.

The purpose of this model is to qualify the functional behavior of NISP (only the nominal side; no redundancy), to perform a conducted susceptibility test at cold operational temperature and to prepare the full NISP TV / performance to be done on the NISP FM.

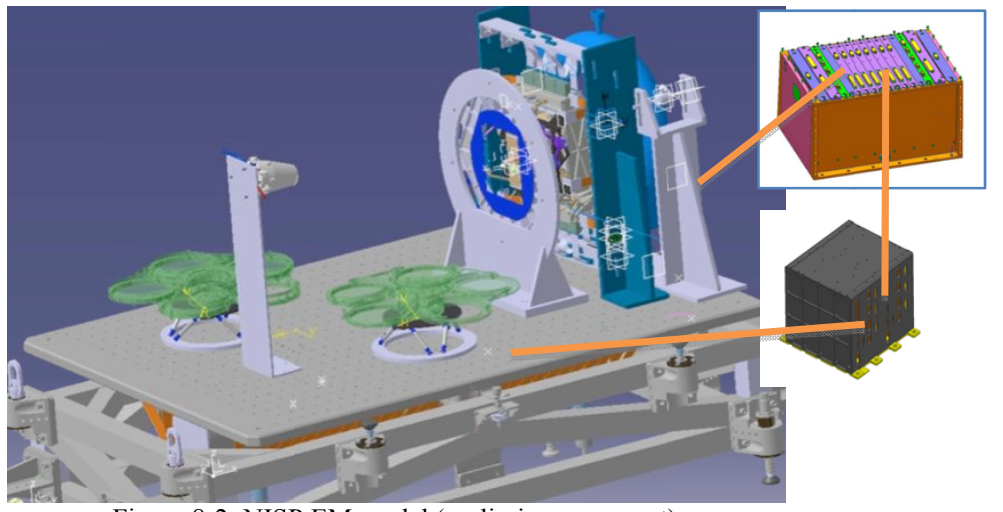

Figure 8-2: NISP EM model (preliminary concept)

At the end of EM TV test, the DPU and the ICU will be delivered to ESA as the Avionic Model (AVM) to be delivered to ESA in September 2017. NIOMA and NI-DS electrical simulators will be also provided.

The NISP FM model:

Is expected to be delivered to ESA before mid 2018. 


\section{ACKNOWLEDGMENTS}

We thank ALL the funding agencies of the NISP project: CNES, ASI, DLR, ERDF, MINECO, Norway space agency, Denmark space agency, ESA, NASA and ALL institutes participating to this project.

\section{REFERENCES}

[1] G. Racca, et al, "The Euclid mission design", Proc. SPIE 9904-19 (2016)

[2] T. Pamplona, et al, "Silicon Carbide main structure for Euclid NISP Instrument in final development", Proc. SPIE 9912, Space Telescopes and Instrumentation 2016: Optical, Infrared, and Millimeter Wave, 9912-21 (2016)

[3] A. Costille, et al, "Final design and choices for EUCLID NISP grism", Proc. SPIE 9912, Space Telescopes and Instrumentation 2016: Optical, Infrared, and Millimeter Wave, 9912-82 (2016)

[4] B. Kubik, et al, "Low noise flux estimate and data quality control monitoring in EUCLID-NISP cosmological survey", Proc. SPIE TBD (2016)

[5] A. Secroun, et al, "Characterization of H2RG IR detectors for the Euclid NISP instrument", Proc. SPIE TBD (2016)

[6] J.C Clemens, et al, "EUCLID detector system demonstrator model: a first demonstration of the NISP detection system", Proc. SPIE 9602, UV/Optical/IR Space Telescopes and Instruments: Innovative Technologies and Concepts VII (September 2015)

[7] S. Ligori, et al., "Detailed design and first tests of the application software for the instrument control unit of Euclid-NISP", SPIE Conference Series, Vol. 9904, "Space Telescopes and Instrumentation 2016: Optical, Infrared, and Millimeter Wave" (2016)

[8] C.Bonoli, et al, "On-board data processing for the Near Infrared Spectrograph and Photometer instrument (NISP) of the EUCLID mission", Proc. SPIE 9912, Space Telescopes and Instrumentation 2016: Optical, Infrared, and Millimeter Wave 